\title{
Time-space variable-order fractional nonlinear system of thermoelasticity: numerical treatment
}

\author{
Taghreed A. Assiri ${ }^{* *}$
}

\section{"Correspondence:}

taassiri@uqu.edu.sa;

rieda2008@gmail.com

'Department of Mathematics,

Faculty of Science, Umm Al-Qura

University, Mecca, Saudi Arabia

\section{Springer}

\begin{abstract}
This paper focuses on a numerical study of the general time-space variable-order fractional nonlinear problem of thermoelasticity in one dimension using the weighted average nonstandard finite difference (WANSFD). By replacing the second order space derivative with a Riesz fractional variable-order derivative and the time derivative by Caputo fractional variable-order operator in the standard system which arises in thermoelasticity, we obtain this general system. Using a kind of John von Neumann technique, we study the stability of the designed schemes. Also, the truncation error of the introduced schemes is studied. Our numerical treatment is shown graphically. These results expose that WANSFD approach is suitable and effective for solving the proposed system; moreover, it is easy to implement.

Keywords: Time-space variable-order fractional nonlinear thermoelasticity; Riesz fractional fractional variable-order derivative; John von Neumann stability method; Weighted average nonstandard finite difference methods
\end{abstract}

\section{Introduction}

Today scientists in different fields such as plasma waves, fluid mechanics systems and solid state physics use coupled partial differential equations to describe many phenomena. A system of nonlinear coupled hyperbolic and parabolic equations is always used in studies of circled fuel reactor, radiation hydrodynamics, magnetoelasticity, thermoelasticity, and in biology [1-5]. It is known that studying the behavior of solutions of these systems is a very important and difficult area of research. A lot of authors [4-6] have been studying the existence of solutions for such models, as well as their uniqueness and stability.

It is very difficult to obtain the analytic exact solutions of such nonlinear problems and, unfortunately, only in simple cases one can find these solutions. Therefore, it is very important to approximate them numerically, which requires great potential from the researchers. The authors of [7] and of the references therein studied a system of coupled parabolic equations. Some of these studies depended on the finite element technique $[8,9]$ and on the finite difference technique $[1,2,10]$ for a nonlinear system of coupled hyperbolic and parabolic equations. In [11, 12], the authors apply the Adomian decomposition approach and the variational iteration approach for solving the model. In [3], the authors

(c) The Author(s) 2020. This article is licensed under a Creative Commons Attribution 4.0 International License, which permits use, sharing, adaptation, distribution and reproduction in any medium or format, as long as you give appropriate credit to the original author(s) and the source, provide a link to the Creative Commons licence, and indicate if changes were made. The images or other third party material in this article are included in the article's Creative Commons licence, unless indicated otherwise in a credit line to the material. If material is not included in the article's Creative Commons licence and your intended use is not permitted by statutory regulation or exceeds the permitted use, you will need to obtain permission directly from the copyright holder. To view a copy of this licence, visit http://creativecommons.org/licenses/by/4.0/. 
found the solution of a nonlinear system of coupled hyperbolic and parabolic equations with specified harmonic displacement at the border depending on the Poincaré extension with a small parameter.

It is well known, since the last few years, that the fractional derivative operators are more compatible to describe the properties of many real-life problems and more helpful than those of integer-order to understand such problems [13]. In fact, the next state in the model with a fractional-order derivative is based on all of its prior states and not only on its present state as in the case with a derivative of integer order. And therefore, the derivative of fractional order will be more authentic than that of integer order. Inclusion of the hereditary properties and memory effect of processes in numerous real-life problems is considered as one of the most important advantages of the fractional derivative [14].

The fractional differential equations is the best way to describe the fractional models. These equations are solved by different methods. Some of the introduced numerical methods are: Adomian decomposition technique [15], finite element technique [16], finite difference technique [17, 18], homotopy perturbation technique [19], spectral methods [20, 21], Taylor collocation methods [22], and variational iteration methods [23].

Recently, many physical, mathematical, financial, viscoelasticity, mechanical, and engineering problems have been described by the fractional variable-order pseudodifferential operators, see [24-32]. Also, the underlying differential operators in many dynamic processes appear as fractional and are dynamic in a sense that their derivative order is field-changing, i.e., it may change with space and/or time. Subsequently, a lot of scientists have been studying the characteristic of the variable-order fractional derivatives. In 1993, Samko with others [31] introduced this motivating expansion of the standard fractional derivatives by letting the order of the fractional derivative be not a constant but a function of an independent variable. After that, many authors have proposed various definitions of variable-order fractional derivative to fit coveted aims. Lorenzo et al. in [33] introduced in details the connotation of variable-order derivative and inspected some variable-order definitions. Coimbra [34] suggested a new definition for the variable-order fractional operator by using the Laplace transform of the Caputo fractional derivative.

The nonstandard finite difference methods (NSFDMs) were suggested by Mickens [3537] to improve the discretization of some terms in the studied differential equation. Using a specific discretization and the denominator function, this approach will be more stable and more accurate than the standard approach $[38,39]$, such that the proposed approach is not difficult to build [40]. The workable implementation of the NSFDMs exists in the research areas of chemistry, physics, and engineering [41-43]. In particular, the most fascinating usage is in mathematical ecology and mathematical biology [44, 45], where the advantages of the NSFDMs have been shown markedly. Also, the active keeping property of the NSFDMs is well behaved in solving systems of fractional-order differential equations, like the fractional-order neuron model [46],the fractional-order Rössler model [47], and the Hodgkin-Huxley system with fractional order derivative [48].

In addition, the weighted average finite difference method (WAFDM) [49-51] can depend on the weight factor, and the implicit or explicit method (more stable or easy for coding, respectively).

In this work, we merge between those two techniques to find numerical approximating scheme for a time-space variable-order fractional nonlinear system of coupled hyperbolic and parabolic differential equations. This scheme has the advantages of these two 
approaches and therefore will has a larger stability region and will be more accurate. The introduced method is called the weighted average nonstandard finite difference method (WANSFDM) (see [18, 50], and [52]).

Consider the system of general time-space variable-order fractional nonlinear, coupled, hyperbolic and parabolic partial differential equations in one-dimension with additional terms of a heat supply and a force term in the equation of heat conduction and in the equation of the motion, respectively:

$$
\begin{aligned}
& { }_{0}^{c} D_{t}^{\mu(x, t)} u-a_{1}\left(u_{x}, \theta\right) R^{\alpha(x, t)} u+b_{1}\left(u_{x}, \theta\right) R^{\alpha(x, t)-1} \theta=f(x, t), \quad x \in \mathbb{R}, \\
& { }_{0}^{c} D_{t}^{\mu(x, t)-1} \theta-d_{1}\left(u_{x}, \theta\right) R^{\alpha(x, t)} \theta-h_{1}\left(u_{x x}, \theta\right) R^{\alpha(x, t)-1} \theta+c_{1}\left(u_{x}, \theta\right) u_{x t}=l(x, t), \quad t>0,
\end{aligned}
$$

with initial conditions:

$$
\theta(x, 0)=\theta^{0}, \quad u(x, 0)=u^{0}(x), \quad u_{t}(x, 0)=u^{1}(x)
$$

such that $\theta=\theta(x, t)$ is the difference of the temperature of the body from $t_{0}=0$ and $u=$ $u(x, t)$ is the displacement of body from balance, ${ }_{0}^{c} D_{t}^{\mu(x, t)}$ is the variable-order Caputo time fractional derivative with $1<\mu(x, t) \leq 2, R^{\alpha(x, t)}$ is the Riesz variable-order fractional space derivative with $1<\alpha(x, t) \leq 2, a_{1}, b_{1}, c_{1}, d_{1}$, and $h_{1}$, are given smooth functions and the subscripts denote partial derivatives.

Many articles like [1,53-55] have widely studied this system (1). When $\mu(x, t)=2$ and $\alpha(x, t)=2$, in [3,53] we found specific details of the meaning and application of such this system in physics. Also, in [50] the authors studied this system in case of time fractional derivative only. These works depend on the fundamental researches in which the theory of fractional thermoelasticity was introduced (see [56-58], and [59]).

In reality, the Caputo variable-order fractional derivative operator has many advantages when used to describe the derivative in initial value differential equations. The most important feature of variable-order Caputo's definition is that the conditions of the initial value for the variable-order fractional differential equations with the variable-order $\mathrm{Ca}$ puto derivatives will be taken as in the case of differential equations of integer order, therefore, the time variable-order fractional derivatives are often taken in the Caputo sense. Also, it is usually to define the space variable-order fractional derivative as the Riesz variable-order fractional operator. The author in [13] concluded that "the complete theory of fractional differential equations, especially the theory of boundary value problems for fractional differential equations, can be developed only with the use of both left and right derivatives." So, in this paper all the spatial derivatives are Riesz variable-order fractional derivatives, which include the right and left variable-order Riemann-Liouville fractional operators.

The fundamental aim of the present article is to carry out numerical treatment of the time-space variable-order fractional differential model (1) which arises in fractional variable-order thermoelasticity using the WANSFD technique. In reality, there are no studies in the literature using WANSFD technique to solve similar systems with variableorder fractional derivatives, so our attention to such an approach is motivated.

The article is arranged as follows: In the next section, we remind the elementary facts of the used methods (NSFDMs) and present many definitions of the derivatives of fractional variable order. In Sect. 3, in three different cases, we propose WANSFD schemes 
for the time-space variable order fractional model (1). We analyze, in Sect. 4, the stability of the suggested schemes and discuss their truncation error. Section 5 is devoted to some numerical consequences which are reported to exhibit the accuracy and the efficiency of the offered technique. Finally, in Sect. 6, we give conclusions of this work.

\section{Preliminaries and definitions}

\subsection{The nonstandard finite difference technique}

The NSFDMs were suggested firstly by Mickens [35-37]. These techniques construct an approximating discrete schemes for partial differential equations (PDEs) or ordinary differential equations (ODEs). NSFDMs can preserve the properties of the exact solution of the studied PDEs or ODEs depending on the following principles [36]:

1. In general, the denominator functions for the discrete derivatives must be expressed in terms of the step sizes.

2. The orders of the discrete derivatives should be equal to the orders of the corresponding derivatives of the differential equations.

3. The nonlinear terms should be approximated in a nonlocal approximation manner.

4. The schemes must not have solutions which do not coincide with solutions of the studied differential models.

5. Specific conditions which keep the analytic solutions of the studied differential equations must also be specific discrete conditions for a scheme of the finite difference.

In brief, for approximating $\frac{d y}{d t}$ using the Euler approach, we usually depend on $\frac{y(t+h)-y(t)}{h}$ but here we will use $\frac{y(t+h)-y(t)}{\phi(h)}$ instead, where $\phi(h)$ is a continuous function of step size $h$ which satisfies the following conditions:

$$
\phi(h)=h+O\left(h^{2}\right), \quad 0<\phi(h)<1, h \rightarrow 0 .
$$

Furthermore, if there is a nonlinear term in the studied differential equation, we replace it in a nonlocal manner as, for example,

$$
x y \rightarrow\left\{\begin{array}{l}
x_{n-1} y_{n}, \\
x_{n+1} y_{n}, \\
x_{n} y_{n+1} .
\end{array}\right.
$$

\subsection{Fractional variable-order calculus definitions}

In the literature, there are a lot of definitions of the operators of fractional variable-order derivatives (see, e.g., [60-63]). The space-fractional variable-order derivatives are usually given by the Riesz fractional variable-order derivatives. As for the time-fractional derivatives, they are usually defined in the Grünwald-Letnikov, Riemann-Liouville, or Caputo sense.

Definition 2.1 ([28]) Let $\alpha(t) \in \mathbb{R}^{+}$. The variable-order Caputo fractional derivative of order $\alpha(t)$ is defined by

$$
\left({ }_{0}^{c} D_{t}^{\alpha(t)} f\right)(t)=\frac{1}{\Gamma(n-\alpha(t))} \int_{0}^{t} \frac{f^{(n)}(\xi)}{(t-\xi)^{1-n+\alpha(t)}} d \xi, \quad t>0,
$$

where $f(t) \in C^{n}[0, \infty[, n=[\alpha(t)]+1$. 
Definition 2.2 ([28]) The right- and left-sided variable-order Riemann-Liouville operators of fractional derivatives where $t \in \mathbb{R}$ and $\alpha(t) \in \mathbb{R}^{+}, n=[\alpha(t)]+1$ are

$$
\begin{aligned}
& \left({ }_{t} D_{b}^{\alpha(t)} f\right)(t)=\frac{(-1)^{n}}{\Gamma(n-\alpha(t))}\left(\frac{d}{d t}\right)^{n} \int_{t}^{b} \frac{f(\xi)}{(\xi-t)^{1+\alpha(t)-n}} d \xi \\
& \left({ }_{a} D_{t}^{\alpha(t)} f\right)(t)=\frac{1}{\Gamma(n-\alpha(t))}\left(\frac{d}{d t}\right)^{n} \int_{a}^{t} \frac{f(\xi)}{(t-\xi)^{1+\alpha(t)-n}} d \xi .
\end{aligned}
$$

Definition 2.3 ([28]) The right- and left-sided variable-order Grünwald-Letnikov fractional derivatives are given by

$$
\begin{aligned}
& D_{b-}^{\alpha(x)} f(t)=\lim _{h \rightarrow 0, n \tau=b-t} h^{-\alpha(t)} \sum_{j=0}^{n}(-1)^{j}\left(\begin{array}{c}
\alpha(t) \\
j
\end{array}\right) f(t+j h), \\
& D_{a+}^{\alpha(x)} f(t)=\lim _{h \rightarrow 0, n \tau=t-a} h^{-\alpha(t)} \sum_{j=0}^{n}(-1)^{j}\left(\begin{array}{c}
\alpha(t) \\
j
\end{array}\right) f(t-j h),
\end{aligned}
$$

where $[x]$ denotes the integer part of $x$ and $h$ is the step size.

Definition 2.4 ([30]) The variable order Riesz fractional derivative is given by

$$
R^{\alpha(t)} f(t)=-\frac{1}{2 \cos \left(\frac{\pi \alpha(t)}{2}\right)}\left({ }_{a} D_{t}^{\alpha(t)}+{ }_{t} D_{b}^{\alpha(t)}\right) f(t) .
$$

\section{Structure of the WANSFD for the proposed system}

In the current part of this paper, we introduce WANSFDM for obtaining a discrete scheme for the system (1). Here, it is valuable to remind that the distinction between "the weighted average nonstandard finite difference technique" and "the weighted average standard finite difference technique" is analogous to the distinction between the nonstandard finite difference technique and the finite difference technique. The problem of finding the numerical solution of the system (1) is in approximating the fractional variable-order Caputo and Riemann-Liouville variable-order derivatives by WANSFD scheme when $\sigma \in[0,1]$ is the weight factor.

Let $N, M \in \mathbb{N}$ and consider

$$
x_{n}=n h, \quad n=0,1,2, \ldots, N, \quad t_{m}=m \triangle t, \quad m=0,1,2, \ldots, M,
$$

the coordinates of the mesh points, where

$$
\Delta t=T / M, \quad h=(b-a) / N .
$$

We will denote, at a grid point $\left(x_{n}, t_{m}\right)=(n h, m \triangle t)$, the approximated values of $u, \theta$ by $u_{n}^{m}, \theta_{n}^{m}$, respectively. The nonstandard difference approximation of variable-order Caputo derivative is introduced in the form:

$$
\begin{aligned}
& { }_{0}^{c} D_{t}^{\mu(x, t)} u(x, t) \\
& \quad=\frac{1}{\Gamma(2-\mu(x, t))} \int_{0}^{t} \frac{1}{(t-\tau)^{(\mu(x, t)-1)}} \frac{\partial^{2} u(x, \tau)}{\partial \tau^{2}} d \tau
\end{aligned}
$$




$$
\begin{aligned}
= & \frac{1}{\Gamma(2-\mu(x, t))} \sum_{0}^{m-1} \int_{j \Delta t}^{(j+1) \Delta t} z^{(1-\mu(x, t))} \frac{\partial^{2} u(x, t-z)}{\partial z^{2}} d z \\
= & \frac{1}{\Gamma(2-\mu(x, t))} \sum_{0}^{m-1} \frac{u(x, t-(j-1) \Delta t)-2 u(x, t-j \Delta t)+u(x, t-(j+1) \Delta t)}{(\varphi(\Delta t))^{2}} \\
& \times \int_{j \Delta t}^{(j+1) \Delta t} z^{(1-\mu(x, t))} d z \\
= & \frac{(\triangle t)^{2-\mu(x, t)}}{\Gamma(3-\mu(x, t))(\varphi(\Delta t))^{2}} \\
& \times \sum_{0}^{m-1}[u(x, t-(j-1) \triangle t)-2 u(x, t-j \triangle t)+u(x, t-(j+1) \Delta t)] \\
& \times\left[(j+1)^{2-\mu(x, t)}-j^{2-\mu(x, t)}\right] .
\end{aligned}
$$

Similarly,

$$
\begin{aligned}
{ }_{0}^{c} D_{t}^{\mu(x, t)-1} \theta(x, t)= & \frac{(\triangle t)^{2-\mu(x, t)}}{2 \Gamma(3-\mu(x, t))(\varphi(\triangle t))} \sum_{0}^{m-1}[\theta(x, t-(j-1) \triangle t)-\theta(x, t-(j+1) \triangle t)] \\
& \times\left[(j+1)^{2-\mu(x, t)}-j^{2-\mu(x, t)}\right] .
\end{aligned}
$$

Also, the approximations of variable-order Riemann-Liouville derivative using shifted Grünwald-Letnikov variable-order fractional derivative [29] are given as follows:

$$
\begin{aligned}
& \left({ }_{a} D_{x}^{\alpha(x, t)} u(x, t)\right)_{n}^{m}=h^{-\alpha_{n+1}^{m}} \sum_{j=0}^{n+1} g_{j}^{\alpha_{n+1}^{m}} u_{n+1-j}^{m}, \\
& \left({ }_{x} D_{b}^{\alpha(x, t)} u\right)(x, t)_{n}^{m}=h^{-\alpha_{n+1}^{m}} \sum_{j=0}^{N-n+1} g_{j}^{\alpha_{n-1}^{m}} u_{n-1+j}^{m},
\end{aligned}
$$

where

$$
g_{j}^{\alpha_{n}^{m}}=(-1)^{j}\left(\begin{array}{c}
\alpha_{n}^{m} \\
j
\end{array}\right) .
$$

In this paper we study the proposed model in three different cases:

1. $a_{1}(x, t)=1+2 \gamma u_{x}+3 \delta u_{x}^{2}-\beta_{2} \theta, b_{1}(x, t)=-\left(\beta_{1}+\beta_{2} u_{x}\right), c_{1}(x, t)=-a-b u_{x}, d_{1}(x, t)=$ $1+\alpha^{*} u_{x}$, and $h_{1}(x, t)=\alpha^{*} u_{x x}$ (as in [1]) such that the constants have specific orders as follows:

$$
\begin{aligned}
& \delta=O\left(1 \text { to } 10^{-1}\right), \quad \gamma=O(1), \quad \beta_{1}=O\left(10^{-3}\right), \quad \beta_{2}=O\left(10^{-3}\right), \\
& \alpha^{*}=O(1), \quad a=O\left(10^{-1}\right), \quad b=O\left(10^{-1}\right),
\end{aligned}
$$

therefore system(1) can be written as follows:

$$
\begin{aligned}
& { }_{0}^{c} D_{t}^{\mu(x, t)} u-\left(1+2 \gamma u_{x}+3 \delta u_{x}^{2}-\beta_{2} \theta\right) R^{\alpha(x, t)} u-\left(\beta_{1}+\beta_{2} u_{x}\right) R^{\alpha(x, t)-1} \theta \\
& \quad=f(x, t), \quad x \in \mathbb{R}, \\
& { }_{0}^{c} D_{t}^{\mu(x, t)-1} \theta-\left(1+\alpha^{*} u_{x}\right) R^{\alpha(x, t)} \theta-\alpha^{*} u_{x x} R^{\alpha(x, t)-1} \theta+\left(-a-b u_{x}\right) u_{x t}=l(x, t), \quad t>0,
\end{aligned}
$$


Assiri Advances in Difference Equations

(2020) 2020:288

Page 7 of 27

depending on the following difference approximations

$$
\begin{aligned}
&\left({ }_{0}^{c} D_{t}^{\mu(x, t)} u(x, t)\right)_{n}^{m}= \frac{(\triangle t)^{2-\mu_{n}^{m}}}{\Gamma\left(3-\mu_{n}^{m}\right)(\varphi(\Delta t))^{2}} \sum_{0}^{m-1}\left[u_{n}^{m-j+1}-2 u_{n}^{m-j}+u_{n}^{m-j-1}\right] \\
& \times\left[(j+1)^{2-\mu_{n}^{m}}-j^{2-\mu_{n}^{m}}\right]+O\left((\varphi(\Delta t))^{2}\right) \\
&\left({ }_{0}^{c} D_{t}^{\mu(x, t)-1} \theta(x, t)\right)_{n}^{m}= \frac{(\triangle t)^{2-\mu_{n}^{m}}}{2 \Gamma\left(3-\mu_{n}^{m}\right)(\varphi(\Delta t))} \sum_{0}^{m-1}\left[\theta_{n}^{m-j+1}-\theta_{n}^{m-j-1}\right]\left[(j+1)^{2-\mu_{n}^{m}}-j^{2-\mu_{n}^{m}}\right] \\
&+O\left((\varphi(\triangle t))^{2}\right), \\
&\left(R^{\alpha(x, t)} u(x, t)\right)_{n}^{m}=- \frac{(\phi(h))^{-\alpha_{n+1}^{m}}}{2 \cos \left(\frac{\pi \alpha_{n}^{m}}{2}\right)}\left(\sum_{j=0}^{n+1} g_{j}^{\alpha_{n+1}^{m}} u_{n+1-j}^{m}+\sum_{j=0}^{N-n+1} g_{j}^{\alpha_{n-1}^{m}} u_{n-1+j}^{m}\right) \\
&\left(R^{\alpha(x, t)-1} u(x, t)\right)_{n}^{m}=-\frac{(\phi(h))^{-\alpha_{n+1}^{m}-1}}{2 \cos \left(\frac{\pi \alpha_{n}^{m}-1}{2}\right)}\left(\sum_{j=0}^{n+1} g_{j}^{\alpha_{n+1}^{m}-1} u_{n+1-j}^{m}+\sum_{j=0}^{N-n+1} g_{j}^{\alpha_{n-1}^{m}-1} u_{n-1+j}^{m}\right) \\
&\left(u_{x t}\right)_{n}^{m}=\frac{u_{n+1}^{m+1}-u_{n+1}^{m-1}-u_{n-1}^{m+1}+u_{n-1}^{m-1}}{4(\phi(h)) \cdot \varphi(\triangle t)}+O\left((\varphi(\Delta t))^{2}+(\phi(h))^{2}\right), \\
&\left(u_{x}\right)_{n}^{m}=\frac{u_{n+1}^{m}-u_{n-1}^{m}}{2(\phi(h))}+O\left((\phi(h))^{2}\right),
\end{aligned}
$$

then system (10) takes the following form:

$$
\begin{aligned}
\frac{(\Delta t)^{2-\mu_{n}^{m}}}{\Gamma\left(3-\mu_{n}^{m}\right)(\varphi(\triangle t))^{2}} \sum_{0}^{m-1}\left[u_{n}^{m-j+1}-2 u_{n}^{m-j}+u_{n}^{m-j-1}\right]\left[(j+1)^{2-\mu_{n}^{m}}-j^{2-\mu_{n}^{m}}\right] \\
+\sigma\left(1+2 \gamma \frac{u_{n+1}^{m}-u_{n-1}^{m}}{2(\phi(h))}+3 \delta\left(\frac{u_{n+1}^{m}-u_{n-1}^{m}}{2(\phi(h))}\right)^{2}-\beta_{2} \theta_{n}^{m}\right) \\
\times \frac{(\phi(h))^{-\alpha_{n+1}^{m}}}{2 \cos \left(\frac{\pi \alpha_{n}^{m}}{2}\right)}\left(\sum_{j=0}^{n+1} g_{j}^{\alpha_{n+1}^{m}} u_{n+1-j}^{m}+\sum_{j=0}^{N-n+1} g_{j}^{\alpha_{n-1}^{m}} u_{n-1+j}^{m}\right) \\
+(1-\sigma)\left(1+2 \gamma \frac{u_{n+1}^{m+1}-u_{n-1}^{m+1}}{2(\phi(h))}+3 \delta\left(\frac{u_{n+1}^{m+1}-u_{n-1}^{m+1}}{2(\phi(h))}\right)^{2}-\beta_{2} \theta_{n}^{m+1}\right) \\
\times \frac{(\phi(h))^{-\alpha_{n+1}^{m}}}{2 \cos \left(\frac{\pi \alpha_{n}^{m}}{2}\right)}\left(\sum_{j=0}^{n+1} g_{j}^{\alpha_{n+1}^{m+1}} u_{n+1-j}^{m+1}+\sum_{j=0}^{N-n+1} g_{j}^{\alpha_{n-1}^{m+1}} u_{n-1+j}^{m+1}\right) \\
+\sigma\left(\beta_{1}+\beta_{2} \frac{u_{n+1}^{m}-u_{n-1}^{m}}{2(\phi(h))}\right) \\
\times \\
\times \frac{(\phi(h))^{-\alpha_{n+1}^{m}-1}}{2 \cos \left(\frac{\pi \alpha_{n}^{m}-1}{2}\right)}\left(\sum_{j=0}^{n+1} g_{j}^{\alpha_{n+1}^{m}-1} \theta_{n+1-j}^{m}+\sum_{j=0}^{N-n+1} g_{j}^{\alpha_{n-1}^{m}-1} \theta_{n-1+j}^{m}\right) \\
+(1-\sigma)\left(\beta_{1}^{m}+\beta_{2} \frac{u_{n+1}^{m}-u_{n-1}^{m}}{2(\phi(h))}\right) \\
\quad \frac{(\phi(h))^{-\alpha_{n+1}^{m+1}-1}}{2 \cos \left(\frac{\pi \alpha_{n}^{m+1}-1}{2}\right)}\left(\sum_{j=0}^{n+1} g_{j}^{\alpha_{n+1}^{m+1}-1} \theta_{n+1-j}^{m+1}+\sum_{j=0}^{N-n+1} g_{j}^{\alpha_{n-1}^{m+1}-1} \theta_{n-1+j}^{m+1}\right)
\end{aligned}
$$




$$
\begin{aligned}
& \frac{(\triangle t)^{2-\mu_{n}^{m}}}{2 \Gamma\left(3-\mu_{n}^{m}\right)(\varphi(\triangle t))} \sum_{0}^{m-1}\left[\theta_{n}^{m-j+1}-\theta_{n}^{m-j-1}\right]\left[(j+1)^{2-\mu_{n}^{m}}-j^{2-\mu_{n}^{m}}\right] \\
& +\sigma\left(1+\alpha^{*} \frac{u_{n+1}^{m}-u_{n-1}^{m}}{2(\phi(h))}\right) \\
& \times \frac{(\phi(h))^{-\alpha_{n+1}^{m}}}{2 \cos \left(\frac{\pi \alpha_{n}^{m}}{2}\right)}\left(\sum_{j=0}^{n+1} g_{j}^{\alpha_{n+1}^{m}} \theta_{n+1-j}^{m}+\sum_{j=0}^{N-n+1} g_{j}^{\alpha_{n-1}^{m}} \theta_{n-1+j}^{m}\right) \\
& +(1-\sigma)\left(1+\alpha^{*} \frac{u_{n+1}^{m+1}-u_{n-1}^{m+1}}{2(\phi(h))}\right) \\
& \times \frac{(\phi(h))^{-\alpha_{n+1}^{m+1}}}{2 \cos \left(\frac{\pi \alpha_{n}^{m}}{2}\right)}\left(\sum_{j=0}^{n+1} g_{j}^{\alpha_{n+1}^{m+1}} \theta_{n+1-j}^{m+1}+\sum_{j=0}^{N-n+1} g_{j}^{\alpha_{n-1}^{m+1}} \theta_{n-1+j}^{m+1}\right) \\
& +\sigma \alpha^{*} \frac{u_{n+1}^{m}-2 u_{n}^{m}+u_{n-1}^{m}}{(\phi(h))^{2}} \\
& \times \frac{(\phi(h))^{-\alpha_{n+1}^{m}-1}}{2 \cos \left(\frac{\pi \alpha_{n}^{m}-1}{2}\right)}\left(\sum_{j=0}^{n+1} g_{j}^{\alpha_{n+1}^{m}-1} \theta_{n+1-j}^{m}+\sum_{j=0}^{N-n+1} g_{j}^{\alpha_{n-1}^{m}-1} \theta_{n-1+j}^{m}\right) \\
& +(1-\sigma) \alpha^{*} \frac{u_{n+1}^{m+1}-2 u_{n}^{m+1}+u_{n-1}^{m+1}}{(\phi(h))^{2}} \\
& \times \frac{(\phi(h))^{-\alpha_{n+1}^{m+1}-1}}{2 \cos \left(\frac{\pi \alpha_{n}^{m+1}-1}{2}\right)}\left(\sum_{j=0}^{n+1} g_{j}^{\alpha_{n+1}^{m+1}-1} \theta_{n+1-j}^{m+1}+\sum_{j=0}^{N-n+1} g_{j}^{\alpha_{n-1}^{m+1}-1} \theta_{n-1+j}^{m+1}\right) \\
& +\left(-a-b \frac{u_{n+1}^{m}-u_{n-1}^{m}}{2(\phi(h))}\right) \frac{u_{n+1}^{m+1}-u_{n+1}^{m-1}-u_{n-1}^{m+1}+u_{n-1}^{m-1}}{4 \varphi(\triangle t) \cdot(\phi(h))} \\
& -\sigma l_{n}^{m}+(1-\sigma) l_{n}^{m+1}=T_{n}^{m} .
\end{aligned}
$$

The previous substitution gives an error, truncating error, symbolized here by $T_{n}^{m}$. Its estimation will be discussed in Sect. 4.2. After dropping this truncating error, we have the following computable difference scheme:

$$
\begin{aligned}
& \frac{(\triangle t)^{2-\mu_{n}^{m}}}{\Gamma\left(3-\mu_{n}^{m}\right)(\varphi(\triangle t))^{2}} \sum_{0}^{m-1}\left[u_{n}^{m-j+1}-2 u_{n}^{m-j}+u_{n}^{m-j-1}\right]\left[(j+1)^{2-\mu_{n}^{m}}-j^{2-\mu_{n}^{m}}\right] \\
& +\sigma\left(1+2 \gamma \frac{u_{n+1}^{m}-u_{n-1}^{m}}{2(\phi(h))}+3 \delta\left(\frac{u_{n+1}^{m}-u_{n-1}^{m}}{2(\phi(h))}\right)^{2}-\beta_{2} \theta_{n}^{m}\right) \\
& \times \frac{(\phi(h))^{-\alpha_{n+1}^{m}}}{2 \cos \left(\frac{\pi \alpha_{n}^{m}}{2}\right)}\left(\sum_{j=0}^{n+1} g_{j}^{\alpha_{n+1}^{m}} u_{n+1-j}^{m}+\sum_{j=0}^{N-n+1} g_{j}^{\alpha_{n-1}^{m}} u_{n-1+j}^{m}\right) \\
& +(1-\sigma)\left(1+2 \gamma \frac{u_{n+1}^{m+1}-u_{n-1}^{m+1}}{2(\phi(h))}+3 \delta\left(\frac{u_{n+1}^{m+1}-u_{n-1}^{m+1}}{2(\phi(h))}\right)^{2}-\beta_{2} \theta_{n}^{m+1}\right) \\
& \times \frac{(\phi(h))^{-\alpha_{n+1}^{m}}}{2 \cos \left(\frac{\pi \alpha_{n}^{m}}{2}\right)}\left(\sum_{j=0}^{n+1} g_{j}^{\alpha_{n+1}^{m+1}} u_{n+1-j}^{m+1}+\sum_{j=0}^{N-n+1} g_{j}^{\alpha_{n-1}^{m+1}} u_{n-1+j}^{m+1}\right) \\
& +\sigma\left(\beta_{1}+\beta_{2} \frac{u_{n+1}^{m}-u_{n-1}^{m}}{2(\phi(h))}\right)
\end{aligned}
$$




$$
\begin{aligned}
& \times \frac{(\phi(h))^{-\alpha_{n+1}^{m}-1}}{2 \cos \left(\frac{\pi \alpha_{n}^{m}-1}{2}\right)}\left(\sum_{j=0}^{n+1} g_{j}^{\alpha_{n+1}^{m}-1} \theta_{n+1-j}^{m}+\sum_{j=0}^{N-n+1} g_{j}^{\alpha_{n-1}^{m}-1} \theta_{n-1+j}^{m}\right) \\
& +(1-\sigma)\left(\beta_{1}+\beta_{2} \frac{u_{n+1}^{m}-u_{n-1}^{m}}{2(\phi(h))}\right) \\
& \times \frac{(\phi(h))^{-\alpha_{n+1}^{m+1}-1}}{2 \cos \left(\frac{\pi \alpha_{n}^{m+1}-1}{2}\right)}\left(\sum_{j=0}^{n+1} g_{j}^{\alpha_{n+1}^{m+1}-1} \theta_{n+1-j}^{m+1}+\sum_{j=0}^{N-n+1} g_{j}^{\alpha_{n-1}^{m+1}-1} \theta_{n-1+j}^{m+1}\right) \\
& -\sigma f_{n}^{m}+(1-\sigma) f_{n}^{m+1}=0, \\
& \frac{(\triangle t)^{2-\mu_{n}^{m}}}{2 \Gamma\left(3-\mu_{n}^{m}\right)(\varphi(\triangle t))} \sum_{0}^{m-1}\left[\theta_{n}^{m-j+1}-\theta_{n}^{m-j-1}\right]\left[(j+1)^{2-\mu_{n}^{m}}-j^{2-\mu_{n}^{m}}\right] \\
& +\sigma\left(1+\alpha^{*} \frac{u_{n+1}^{m}-u_{n-1}^{m}}{2(\phi(h))}\right) \\
& \times \frac{(\phi(h))^{-\alpha_{n+1}^{m}}}{2 \cos \left(\frac{\pi \alpha_{n}^{m}}{2}\right)}\left(\sum_{j=0}^{n+1} g_{j}^{\alpha_{n+1}^{m}} \theta_{n+1-j}^{m}+\sum_{j=0}^{N-n+1} g_{j}^{\alpha_{n-1}^{m}} \theta_{n-1+j}^{m}\right) \\
& +(1-\sigma)\left(1+\alpha^{*} \frac{u_{n+1}^{m+1}-u_{n-1}^{m+1}}{2(\phi(h))}\right) \\
& \times \frac{(\phi(h))^{-\alpha_{n+1}^{m+1}}}{2 \cos \left(\frac{\pi \alpha_{n}^{m}}{2}\right)}\left(\sum_{j=0}^{n+1} g_{j}^{\alpha_{n+1}^{m+1}} \theta_{n+1-j}^{m+1}+\sum_{j=0}^{N-n+1} g_{j}^{\alpha_{n-1}^{m+1}} \theta_{n-1+j}^{m+1}\right) \\
& +\sigma \alpha^{*} \frac{u_{n+1}^{m}-2 u_{n}^{m}+u_{n-1}^{m}}{(\phi(h))^{2}} \\
& \times \frac{(\phi(h))^{-\alpha_{n+1}^{m}-1}}{2 \cos \left(\frac{\pi \alpha_{n}^{m}-1}{2}\right)}\left(\sum_{j=0}^{n+1} g_{j}^{\alpha_{n+1}^{m}-1} \theta_{n+1-j}^{m}+\sum_{j=0}^{N-n+1} g_{j}^{\alpha_{n-1}^{m}-1} \theta_{n-1+j}^{m}\right) \\
& +(1-\sigma) \alpha^{*} \frac{u_{n+1}^{m+1}-2 u_{n}^{m+1}+u_{n-1}^{m+1}}{(\phi(h))^{2}} \\
& \times \frac{(\phi(h))^{-\alpha_{n+1}^{m+1}-1}}{2 \cos \left(\frac{\pi \alpha_{n}^{m+1}-1}{2}\right)}\left(\sum_{j=0}^{n+1} g_{j}^{\alpha_{n+1}^{m+1}-1} \theta_{n+1-j}^{m+1}+\sum_{j=0}^{N-n+1} g_{j}^{\alpha_{n+1}^{m+1}-1} \theta_{n-1+j}^{m+1}\right) \\
& +\left(-a-b \frac{u_{n+1}^{m}-u_{n-1}^{m}}{2(\phi(h))}\right) \frac{u_{n+1}^{m+1}-u_{n+1}^{m-1}-u_{n-1}^{m+1}+u_{n-1}^{m-1}}{4 \varphi(\triangle t) \cdot(\phi(h))} \\
& -\sigma l_{n}^{m}+(1-\sigma) l_{n}^{m+1}=0 .
\end{aligned}
$$

2. $a_{1}(x, t)=d_{1}(x, t)=1, b_{1}(x, t)=c_{1}(x, t)=\theta u_{x}$, and $h_{1}(x, t)=0$ (as in [55]) so model (1) has the following form:

$$
\begin{aligned}
& { }_{0}^{c} D_{t}^{\mu(x, t)} u-R^{\alpha(x, t)} u+\theta u_{x} R^{\alpha(x, t)-1} \theta=f(x, t), \\
& { }_{0}^{c} D_{t}^{\mu(x, t)-1} \theta-R^{\alpha(x, t)} \theta+\theta u_{x} u_{x t}=l(x, t),
\end{aligned}
$$

and then, using the difference approximations (11a)-(11f), system (14) can be written as follows:

$$
\frac{(\triangle t)^{2-\mu_{n}^{m}}}{\Gamma\left(3-\mu_{n}^{m}\right)(\varphi(\triangle t))^{2}} \sum_{0}^{m-1}\left[u_{n}^{m-j+1}-2 u_{n}^{m-j}+u_{n}^{m-j-1}\right]\left[(j+1)^{2-\mu_{n}^{m}}-j^{2-\mu_{n}^{m}}\right]
$$


Assiri Advances in Difference Equations

(2020) 2020:288

Page 10 of 27

$$
\begin{aligned}
& +\sigma \frac{(\phi(h))^{-\alpha_{n+1}^{m}}}{2 \cos \left(\frac{\pi \alpha_{n}^{m}}{2}\right)}\left(\sum_{j=0}^{n+1} g_{j}^{\alpha_{n+1}^{m}} u_{n+1-j}^{m}+\sum_{j=0}^{N-n+1} g_{j}^{\alpha_{n-1}^{m}} u_{n-1+j}^{m}\right) \\
& +(1-\sigma) \frac{(\phi(h))^{-\alpha_{n+1}^{m+1}}}{2 \cos \left(\frac{\left(\alpha_{n}^{m+1}\right.}{2}\right)}\left(\sum_{j=0}^{n+1} g_{j}^{\alpha_{n+1}^{m+1}} u_{n+1-j}^{m+1}+\sum_{j=0}^{N-n+1} g_{j}^{\alpha_{n-1}^{m+1}} u_{n-1+j}^{m+1}\right) \\
& -\sigma \theta_{n}^{m} \frac{u_{n+1}^{m}-u_{n-1}^{m}}{2(\phi(h))} \frac{(\phi(h))^{-\alpha_{n+1}^{m}-1}}{2 \cos \left(\frac{\pi \alpha_{n}^{m}-1}{2}\right)}\left(\sum_{j=0}^{n+1} g_{j}^{\alpha_{n+1}^{m}} \theta_{n+1-j}^{m}+\sum_{j=0}^{N-n+1} g_{j}^{\alpha_{n-1}^{m}-1} \theta_{n-1+j}^{m}\right) \\
& -(1-\sigma) \theta_{n}^{m} \frac{u_{n+1}^{m+1}-u_{n-1}^{m+1}}{2(\phi(h))} \frac{(\phi(h))^{-\alpha_{n+1}^{m+1}-1}}{2 \cos \left(\frac{\pi \alpha_{n}^{m+1}-1}{2}\right)}\left(\sum_{j=0}^{n+1} g_{j}^{\alpha_{n+1}^{m+1}-1} \theta_{n+1-j}^{m+1}+\sum_{j=0}^{N-n+1} g_{j}^{\alpha_{n-1}^{m+1}-1} \theta_{n-1+j}^{m+1}\right) \\
& -\sigma f_{n}^{m}+(1-\sigma) f_{n}^{m+1}=T_{n}^{m}, \\
& \frac{(\Delta t)^{2-\mu_{n}^{m}}}{2 \Gamma\left(3-\mu_{n}^{m}\right)(\varphi(\Delta t))} \sum_{0}^{m-1}\left[\theta_{n}^{m-j+1}-\theta_{n}^{m-j-1}\right]\left[(j+1)^{2-\mu_{n}^{m}}-j^{2-\mu_{n}^{m}}\right] \\
& +\sigma \frac{(\phi(h))^{-\alpha_{n+1}^{m}}}{2 \cos \left(\frac{\pi \alpha_{n}^{m}}{2}\right)}\left(\sum_{j=0}^{n+1} g_{j}^{\alpha_{n+1}^{m}} \theta_{n+1-j}^{m}+\sum_{j=0}^{N-n+1} g_{j}^{\alpha_{n-1}^{m}} \theta_{n-1+j}^{m}\right) \\
& +(1-\sigma) \frac{(\phi(h))^{-\alpha_{n+1}^{m+1}}}{2 \cos \left(\frac{\pi \alpha_{n}^{m+1}}{2}\right)}\left(\sum_{j=0}^{n+1} g_{j}^{\alpha_{n+1}^{m+1}} \theta_{n+1-j}^{m+1}+\sum_{j=0}^{N-n+1} g_{j}^{\alpha_{n-1}^{m+1}} \theta_{n-1+j}^{m+1}\right) \\
& +\theta_{n}^{m} \frac{u_{n+1}^{m}-u_{n-1}^{m}}{2(\phi(h))} \frac{u_{n+1}^{m+1}-u_{n+1}^{m-1}-u_{n-1}^{m+1}+u_{n-1}^{m-1}}{4 \varphi(\triangle t) \cdot(\phi(h))} \\
& -\sigma l_{n}^{m}+(1-\sigma) l_{n}^{m+1}=T_{n}^{m} .
\end{aligned}
$$

Neglecting the truncation error, then we have

$$
\begin{aligned}
& \frac{(\Delta t)^{2-\mu_{n}^{m}}}{\Gamma\left(3-\mu_{n}^{m}\right)(\varphi(\Delta t))^{2}} \sum_{0}^{m-1}\left[u_{n}^{m-j+1}-2 u_{n}^{m-j}+u_{n}^{m-j-1}\right]\left[(j+1)^{2-\mu_{n}^{m}}-j^{2-\mu_{n}^{m}}\right] \\
& +\sigma \frac{(\phi(h))^{-\alpha_{n+1}^{m}}}{2 \cos \left(\frac{\pi \alpha_{n}^{m}}{2}\right)}\left(\sum_{j=0}^{n+1} g_{j}^{\alpha_{n+1}^{m}} u_{n+1-j}^{m}+\sum_{j=0}^{N-n+1} g_{j}^{\alpha_{n-1}^{m}} u_{n-1+j}^{m}\right) \\
& +(1-\sigma) \frac{(\phi(h))^{-\alpha_{n+1}^{m+1}}}{2 \cos \left(\frac{\pi \alpha_{n}^{m+1}}{2}\right)}\left(\sum_{j=0}^{n+1} g_{j}^{\alpha_{n+1}^{m+1}} u_{n+1-j}^{m+1}+\sum_{j=0}^{N-n+1} g_{j}^{\alpha_{n-1}^{m+1}} u_{n-1+j}^{m+1}\right) \\
& -\sigma \theta_{n}^{m} \frac{u_{n+1}^{m}-u_{n-1}^{m}}{2(\phi(h))} \frac{(\phi(h))^{-\alpha_{n+1}^{m}-1}}{2 \cos \left(\frac{\pi \alpha_{n}^{m}-1}{2}\right)}\left(\sum_{j=0}^{n+1} g_{j}^{\alpha_{n+1}^{m}} \theta_{n+1-j}^{m}+\sum_{j=0}^{N-n+1} g_{j}^{\alpha_{n-1}^{m}-1} \theta_{n-1+j}^{m}\right) \\
& -(1-\sigma) \theta_{n}^{m} \frac{u_{n+1}^{m+1}-u_{n-1}^{m+1}}{2(\phi(h))} \frac{(\phi(h))^{-\alpha_{n+1}^{m+1}-1}}{2 \cos \left(\frac{\pi \alpha_{n}^{m+1}-1}{2}\right)}\left(\sum_{j=0}^{n+1} g_{j}^{\alpha_{n+1}^{m+1}-1} \theta_{n+1-j}^{m+1}+\sum_{j=0}^{N-n+1} g_{j}^{\alpha_{n-1}^{m+1}-1} \theta_{n-1+j}^{m+1}\right) \\
& -\sigma f_{n}^{m}+(1-\sigma) f_{n}^{m+1}=0, \\
& \frac{(\Delta t)^{2-\mu_{n}^{m}}}{2 \Gamma\left(3-\mu_{n}^{m}\right)(\varphi(\Delta t))} \sum_{0}^{m-1}\left[\theta_{n}^{m-j+1}-\theta_{n}^{m-j-1}\right]\left[(j+1)^{2-\mu_{n}^{m}}-j^{2-\mu_{n}^{m}}\right] \\
& +\sigma \frac{(\phi(h))^{-\alpha_{n+1}^{m}}}{2 \cos \left(\frac{\pi \alpha_{n}^{m}}{2}\right)}\left(\sum_{j=0}^{n+1} g_{j}^{\alpha_{n+1}^{m}} \theta_{n+1-j}^{m}+\sum_{j=0}^{N-n+1} g_{j}^{\alpha_{n-1}^{m}} \theta_{n-1+j}^{m}\right)
\end{aligned}
$$




$$
\begin{aligned}
& +(1-\sigma) \frac{(\phi(h))^{-\alpha_{n+1}^{m+1}}}{2 \cos \left(\frac{\pi \alpha_{n}^{m+1}}{2}\right)}\left(\sum_{j=0}^{n+1} g_{j}^{\alpha_{n+1}^{m+1}} \theta_{n+1-j}^{m+1}+\sum_{j=0}^{N-n+1} g_{j}^{\alpha_{n-1}^{m+1}} \theta_{n-1+j}^{m+1}\right) \\
& +\theta_{n}^{m} \frac{u_{n+1}^{m}-u_{n-1}^{m}}{2(\phi(h))} \frac{u_{n+1}^{m+1}-u_{n+1}^{m-1}-u_{n-1}^{m+1}+u_{n-1}^{m-1}}{4 \varphi(\triangle t) \cdot(\phi(h))} \\
& -\sigma l_{n}^{m}+(1-\sigma) l_{n}^{m+1}=0 .
\end{aligned}
$$

3. $a_{1}(x, t)=2-\theta u_{x}, b_{1}(x, t)=c_{1}(x, t)=2+\theta u_{x}, d_{1}(x, t)=\theta$, and $h_{1}(x, t)=0$ (as in [12]) so model (1) has the following form:

$$
\begin{aligned}
& { }_{0}^{c} D_{t}^{\mu(x, t)} u-\left(2-\theta u_{x}\right) R^{\alpha(x, t)} u+\left(2+\theta u_{x}\right) R^{\alpha(x, t)-1} \theta=f(x, t), \quad x \in \mathbb{R}, \\
& { }_{0}^{c} D_{t}^{\mu(x, t)-1} \theta-\theta R^{\alpha(x, t)} \theta+\left(2+\theta u_{x}\right) u_{x t}=l(x, t), \quad t>0,
\end{aligned}
$$

where, depending on the difference approximations (11a)-(11f), model (17) then takes the form:

$$
\begin{aligned}
& \frac{(\triangle t)^{2-\mu_{n}^{m}}}{\Gamma\left(3-\mu_{n}^{m}\right)(\varphi(\triangle t))^{2}} \sum_{0}^{m-1}\left[u_{n}^{m-j+1}-2 u_{n}^{m-j}+u_{n}^{m-j-1}\right]\left[(j+1)^{2-\mu_{n}^{m}}-j^{2-\mu_{n}^{m}}\right] \\
& +\sigma\left(2-\theta_{n}^{m} \frac{u_{n+1}^{m}-u_{n-1}^{m}}{2(\phi(h))}\right) \frac{(\phi(h))^{-\alpha_{n+1}^{m}}}{2 \cos \left(\frac{\pi \alpha_{n}^{m}}{2}\right)}\left(\sum_{j=0}^{n+1} g_{j}^{\alpha_{n+1}^{m}} u_{n+1-j}^{m}+\sum_{j=0}^{N-n+1} g_{j}^{\alpha_{n-1}^{m}} u_{n-1+j}^{m}\right) \\
& +(1-\sigma)\left(2-\theta_{n}^{m+1} \frac{u_{n+1}^{m+1}-u_{n-1}^{m+1}}{2(\phi(h))}\right) \\
& \times \frac{h^{-\alpha_{n+1}^{m+1}}}{2 \cos \left(\frac{\pi \alpha_{n}^{m+1}}{2}\right)}\left(\sum_{j=0}^{n+1} g_{j}^{\alpha_{n+1}^{m+1}} u_{n+1-j}^{m+1}+\sum_{j=0}^{N-n+1} g_{j}^{\alpha_{n-1}^{m+1}} u_{n-1+j}^{m+1}\right) \\
& -\sigma\left(2+\theta_{n}^{m} \frac{u_{n+1}^{m}-u_{n-1}^{m}}{2(\phi(h))}\right) \frac{(\phi(h))^{-\alpha_{n+1}^{m}-1}}{2 \cos \left(\frac{\pi \alpha_{n}^{m}-1}{2}\right)}\left(\sum_{j=0}^{n+1} g_{j}^{\alpha_{n+1}^{m}-1} \theta_{n+1-j}^{m}+\sum_{j=0}^{N-n+1} g_{j}^{\alpha_{n-1}^{m}-1} \theta_{n-1+j}^{m}\right) \\
& -(1-\sigma)\left(2+\theta_{n}^{m+1} \frac{u_{n+1}^{m+1}-u_{n-1}^{m+1}}{2(\phi(h))}\right) \\
& \times \frac{(\phi(h))^{-\alpha_{n+1}^{m+1}-1}}{2 \cos \left(\frac{\pi \alpha_{n}^{m+1}-1}{2}\right)}\left(\sum_{j=0}^{n+1} g_{j}^{\alpha_{n+1}^{m+1}-1} \theta_{n+1-j}^{m+1}+\sum_{j=0}^{N-n+1} g_{j}^{\alpha_{n-1}^{m+1}-1} \theta_{n-1+j}^{m+1}\right) \\
& -\sigma f_{n}^{m}+(1-\sigma) f_{n}^{m+1}=T_{n}^{m}, \\
& \frac{(\Delta t)^{2-\mu_{n}^{m}}}{2 \Gamma\left(3-\mu_{n}^{m}\right)(\varphi(\triangle t))} \sum_{0}^{m-1}\left[\theta_{n}^{m-j+1}-\theta_{n}^{m-j-1}\right]\left[(j+1)^{2-\mu_{n}^{m}}-j^{2-\mu_{n}^{m}}\right] \\
& +\sigma \theta_{n}^{m} \sigma \frac{(\phi(h))^{-\alpha_{n+1}^{m}}}{2 \cos \left(\frac{\pi \alpha_{n}^{m}}{2}\right)}\left(\sum_{j=0}^{n+1} g_{j}^{\alpha_{n+1}^{m}} \theta_{n+1-j}^{m}+\sum_{j=0}^{N-n+1} g_{j}^{\alpha_{n-1}^{m}} \theta_{n-1+j}^{m}\right) \\
& +(1-\sigma) \theta_{n}^{m+1} \frac{(\phi(h))^{-\alpha_{n+1}^{m+1}}}{2 \cos \left(\frac{\pi \alpha_{n}^{m+1}}{2}\right)}\left(\sum_{j=0}^{n+1} g_{j}^{\alpha_{n+1}^{m+1}} \theta_{n+1-j}^{m+1}+\sum_{j=0}^{N-n+1} g_{j}^{\alpha_{n-1}^{m+1}} \theta_{n-1+j}^{m+1}\right) \\
& +\left(2+\theta_{n}^{m} \frac{u_{n+1}^{m}-u_{n-1}^{m}}{2(\phi(h))}\right) \frac{u_{n+1}^{m+1}-u_{n+1}^{m-1}-u_{n-1}^{m+1}+u_{n-1}^{m-1}}{4 \varphi(\triangle t) \cdot(\phi(h))} \\
& -\sigma l_{n}^{m}+(1-\sigma) l_{n}^{m+1}=T_{n}^{m} .
\end{aligned}
$$


Neglecting the truncation error, then we have

$$
\begin{aligned}
& \frac{(\triangle t)^{2-\mu_{n}^{m}}}{\Gamma\left(3-\mu_{n}^{m}\right)(\varphi(\triangle t))^{2}} \sum_{0}^{m-1}\left[u_{n}^{m-j+1}-2 u_{n}^{m-j}+u_{n}^{m-j-1}\right]\left[(j+1)^{2-\mu_{n}^{m}}-j^{2-\mu_{n}^{m}}\right] \\
& +\sigma\left(2-\theta_{n}^{m} \frac{u_{n+1}^{m}-u_{n-1}^{m}}{2(\phi(h))}\right) \frac{(\phi(h))^{-\alpha_{n+1}^{m}}}{2 \cos \left(\frac{\pi \alpha_{n}^{m}}{2}\right)}\left(\sum_{j=0}^{n+1} g_{j}^{\alpha_{n+1}^{m}} u_{n+1-j}^{m}+\sum_{j=0}^{N-n+1} g_{j}^{\alpha_{n-1}^{m}} u_{n-1+j}^{m}\right) \\
& +(1-\sigma)\left(2-\theta_{n}^{m+1} \frac{u_{n+1}^{m+1}-u_{n-1}^{m+1}}{2(\phi(h))}\right) \\
& \times \frac{h^{-\alpha_{n+1}^{m+1}}}{2 \cos \left(\frac{\pi \alpha_{n}^{m+1}}{2}\right)}\left(\sum_{j=0}^{n+1} g_{j}^{\alpha_{n+1}^{m+1}} u_{n+1-j}^{m+1}+\sum_{j=0}^{N-n+1} g_{j}^{\alpha_{n-1}^{m+1}} u_{n-1+j}^{m+1}\right) \\
& -\sigma\left(2+\theta_{n}^{m} \frac{u_{n+1}^{m}-u_{n-1}^{m}}{2(\phi(h))}\right) \frac{(\phi(h))^{-\alpha_{n+1}^{m}-1}}{2 \cos \left(\frac{\pi \alpha_{n}^{m}-1}{2}\right)}\left(\sum_{j=0}^{n+1} g_{j}^{\alpha_{n+1}^{m}-1} \theta_{n+1-j}^{m}+\sum_{j=0}^{N-n+1} g_{j}^{\alpha_{n-1}^{m}-1} \theta_{n-1+j}^{m}\right) \\
& -(1-\sigma)\left(2+\theta_{n}^{m+1} \frac{u_{n+1}^{m+1}-u_{n-1}^{m+1}}{2(\phi(h))}\right) \\
& \times \frac{(\phi(h))^{-\alpha_{n+1}^{m+1}-1}}{2 \cos \left(\frac{\pi \alpha_{n}^{m+1}-1}{2}\right)}\left(\sum_{j=0}^{n+1} g_{j}^{\alpha_{n+1}^{m+1}-1} \theta_{n+1-j}^{m+1}+\sum_{j=0}^{N-n+1} g_{j}^{\alpha_{n-1}^{m+1}-1} \theta_{n-1+j}^{m+1}\right) \\
& -\sigma f_{n}^{m}+(1-\sigma) f_{n}^{m+1}=0, \\
& \frac{(\triangle t)^{2-\mu_{n}^{m}}}{2 \Gamma\left(3-\mu_{n}^{m}\right)(\varphi(\triangle t))} \sum_{0}^{m-1}\left[\theta_{n}^{m-j+1}-\theta_{n}^{m-j-1}\right]\left[(j+1)^{2-\mu_{n}^{m}}-j^{2-\mu_{n}^{m}}\right] \\
& +\sigma \theta_{n}^{m} \sigma \frac{(\phi(h))^{-\alpha_{n+1}^{m}}}{2 \cos \left(\frac{\pi \alpha_{n}^{m}}{2}\right)}\left(\sum_{j=0}^{n+1} g_{j}^{\alpha_{n+1}^{m}} \theta_{n+1-j}^{m}+\sum_{j=0}^{N-n+1} g_{j}^{\alpha^{m}} \theta_{n-1+j}^{m}\right) \\
& +(1-\sigma) \theta_{n}^{m+1} \frac{(\phi(h))^{-\alpha_{n+1}^{m+1}}}{2 \cos \left(\frac{\pi \alpha_{n}^{m+1}}{2}\right)}\left(\sum_{j=0}^{n+1} g_{j}^{\alpha_{n+1}^{m+1}} \theta_{n+1-j}^{m+1}+\sum_{j=0}^{N-n+1} g_{j}^{\alpha_{n-1}^{m+1}} \theta_{n-1+j}^{m+1}\right) \\
& +\left(2+\theta_{n}^{m} \frac{u_{n+1}^{m}-u_{n-1}^{m}}{2(\phi(h))}\right) \frac{u_{n+1}^{m+1}-u_{n+1}^{m-1}-u_{n-1}^{m+1}+u_{n-1}^{m-1}}{4 \varphi(\triangle t) \cdot(\phi(h))} \\
& -\sigma l_{n}^{m}+(1-\sigma) l_{n}^{m+1}=0 \text {. }
\end{aligned}
$$

All of the above schemes (13), (16), and (19) with their particular initial conditions and boundary conditions form an algebraic system of $2(N+1)(M+1)$ nonlinear equations with $u_{n}^{m}, \theta_{n}^{m}(n=0,1,2, \ldots, N, m=0,1,2, \ldots, M)$ as unknowns. The algebraic system can be solved using Newton's iteration method [64].

The schemes (13), (16), and (19) are explicit for $\sigma=1$, partially implicit for $0<\sigma<1$, fully implicit for $\sigma=0$, and for the special case $\sigma=1 / 2$ therefore we use the Crank-Nicholson scheme [51].

\section{Analysis of the stability and truncation error}

\subsection{Stability analysis}

To discuss the stability of the proposed schemes, we use the von Neumann-type stability technique and, because this technique deals only with linear problems, we have to linearize 
these schemes by supposing that the terms $a_{1}, b_{1}, c_{1}, d_{1}, h_{1}$ are constants and considering $f=l=0$ (free source term) (see [1] and [65] for more details). To start our study analysis, we write system (1) in another form as follows:

$$
\begin{aligned}
& { }_{0}^{c} D_{t}^{\mu(x, t)} u-a_{1} R^{\alpha(x, t)} u+b_{1} R^{\alpha(x, t)-1} \theta=0, \\
& { }_{0}^{c} D_{t}^{\mu(x, t)-1} \theta-d_{1} R^{\alpha(x, t)} \theta-h_{1} R^{\alpha(x, t)-1} \theta+c_{1} u_{x t}=0 .
\end{aligned}
$$

Letting $u_{t}=v$, then we can express:

$$
\begin{aligned}
& { }_{0}^{c} D_{t}^{\mu(x, t)-1} v-a_{1} R^{\alpha(x, t)} u+b_{1} R^{\alpha(x, t)-1} \theta=0, \\
& u_{t}=v, \\
& { }_{0}^{c} D_{t}^{\mu(x, t)-1} \theta-d_{1} R^{\alpha(x, t)} \theta-h_{1} R^{\alpha(x, t)-1} \theta+c_{1} v_{x}=0 .
\end{aligned}
$$

Firstly, we write down system (21) in matrix form as follows:

$$
Y_{10}{ }^{C} D_{t}^{\mu-1} X+Y_{2} R^{\alpha(x, t)} X+Y_{3} R^{\alpha(x, t)-1} X+Y_{4} X_{t}+Y_{5} X_{x}+Y_{6} X=0
$$

where

$$
\begin{aligned}
& \boldsymbol{X}=\left(\begin{array}{l}
u \\
v \\
\theta
\end{array}\right), \quad \boldsymbol{Y}_{\mathbf{1}}=\left(\begin{array}{lll}
0 & 1 & 0 \\
0 & 0 & 0 \\
0 & 0 & 1
\end{array}\right), \\
& \boldsymbol{Y}_{\mathbf{2}}=\left(\begin{array}{ccc}
-a_{1} & 0 & 0 \\
0 & 0 & 0 \\
0 & 0 & -d_{1}
\end{array}\right), \quad \boldsymbol{Y}_{\mathbf{3}}=\left(\begin{array}{ccc}
0 & 0 & b_{1} \\
0 & 0 & 0 \\
0 & 0 & -h_{1}
\end{array}\right), \\
& \boldsymbol{Y}_{\mathbf{4}}=\left(\begin{array}{lll}
0 & 0 & 0 \\
1 & 0 & 0 \\
0 & 0 & 0
\end{array}\right), \quad \boldsymbol{Y}_{\mathbf{5}}=\left(\begin{array}{lll}
0 & 0 & 0 \\
0 & 0 & 0 \\
0 & c_{1} & 0
\end{array}\right), \quad \boldsymbol{Y}_{\mathbf{6}}=\left(\begin{array}{ccc}
0 & 0 & 0 \\
0 & -1 & 0 \\
0 & 0 & 0
\end{array}\right) .
\end{aligned}
$$

Now, we write system (22) using WANSFDM as follows:

$$
\begin{gathered}
\boldsymbol{Y}_{\mathbf{1}} \frac{(\Delta t)^{2-\mu_{n}^{m}}}{2 \Gamma\left(3-\mu_{n}^{m}\right)(\varphi(\Delta t))} \sum_{j=0}^{m-1}\left[\boldsymbol{X}_{n}^{m-j+1}-\boldsymbol{X}_{n}^{m-j-1}\right]\left[(j+1)^{2-\mu_{n}^{m}}-j^{2-\mu_{n}^{m}}\right] \\
-\sigma \boldsymbol{Y}_{\mathbf{2}} \frac{(\phi(h))^{-\alpha_{n+1}^{m}}}{2 \cos \left(\frac{\pi \alpha_{n}^{m}}{2}\right)}\left(\sum_{j=0}^{n+1} g_{j}^{\alpha_{n+1}^{m}} \boldsymbol{X}_{n+1-j}^{m}+\sum_{j=0}^{N-n+1} g_{j}^{\alpha_{n-1}^{m}} \boldsymbol{X}_{n-1+j}^{m}\right) \\
-(1-\sigma) \boldsymbol{Y}_{\mathbf{2}} \frac{(\phi(h))^{-\alpha_{n+1}^{m+1}}}{2 \cos \left(\frac{\pi \alpha_{n}^{m}}{2}\right)}\left(\sum_{j=0}^{n+1} g_{j}^{\alpha_{n+1}^{m+1}} \boldsymbol{X}_{n+1-j}^{m+1}+\sum_{j=0}^{N-n+1} g_{j}^{\alpha_{n-1}^{m+1}} \boldsymbol{X}_{n-1+j}^{m+1}\right) \\
-\sigma \boldsymbol{Y}_{\mathbf{3}} \frac{(\phi(h))^{-\alpha_{n+1}^{m}-1}}{2 \cos \left(\frac{\pi \alpha_{n}^{m}-1}{2}\right)}\left(\sum_{j=0}^{n+1} g_{j}^{\alpha_{n+1}^{m}-1} \boldsymbol{X}_{n+1-j}^{m}+\sum_{j=0}^{N-n+1} g_{j}^{\alpha_{n-1}^{m}-1} \boldsymbol{X}_{n-1+j}^{m}\right)
\end{gathered}
$$




$$
\begin{aligned}
& -(1-\sigma) \boldsymbol{Y}_{\mathbf{3}} \frac{(\phi(h))^{-\alpha_{n+1}^{m+1}-1}}{2 \cos \left(\frac{\pi \alpha_{n}^{m}-1}{2}\right)}\left(\sum_{j=0}^{n+1} g_{j}^{\alpha_{n+1}^{m+1}-1} \boldsymbol{X}_{n+1-j}^{m+1}+\sum_{j=0}^{N-n+1} g_{j}^{\alpha_{n-1}^{m+1}-1} \boldsymbol{X}_{n-1+j}^{m+1}\right) \\
& +\boldsymbol{Y}_{\mathbf{4}} \frac{\boldsymbol{X}_{n}^{m+1}-\boldsymbol{X}_{n}^{m}}{\varphi(\Delta t)} \\
& +\sigma \boldsymbol{Y}_{\mathbf{5}} \frac{\boldsymbol{X}_{n+1}^{m}-\boldsymbol{X}_{n-1}^{m}}{2 \phi(h)}+(1-\sigma) \boldsymbol{Y}_{\mathbf{5}} \frac{\boldsymbol{X}_{n+1}^{m+1}-\boldsymbol{X}_{n-1}^{m+1}}{2 \phi(h)} \\
& +\sigma \boldsymbol{Y}_{\mathbf{6}} \boldsymbol{X}_{n}^{m}+(1-\sigma) \boldsymbol{Y}_{\mathbf{6}} \boldsymbol{X}_{n}^{m+1}=0,
\end{aligned}
$$

which takes the following form:

$$
\begin{aligned}
\boldsymbol{Y}_{\mathbf{1}} \frac{(\Delta t)^{2-\mu_{n}^{m}}}{2 \Gamma\left(3-\mu_{n}^{m}\right)(\varphi(\Delta t))} \boldsymbol{X}_{n}^{m+1}-(1-\sigma) \boldsymbol{Y}_{\mathbf{2}} \frac{(\phi(h))^{-\alpha_{n+1}^{m+1}}}{2 \cos \left(\frac{\pi \alpha_{n}^{m}}{2}\right)}\left(\sum_{j=0}^{N} \widehat{g}_{n, j}^{\alpha_{n+1}^{m+1}} \boldsymbol{X}_{j}^{m+1}\right) \\
-(1-\sigma) \boldsymbol{Y}_{\mathbf{3}} \frac{(\phi(h))^{-\alpha_{n+1}^{m+1}-1}}{2 \cos \left(\frac{\pi \alpha_{n}^{m}-1}{2}\right)}\left(\sum_{j=0}^{N} \widehat{g}_{n, j}^{\alpha_{n+1}^{m+1}-1} \boldsymbol{X}_{j}^{m+1}\right) \\
+\boldsymbol{Y}_{\mathbf{4}} \frac{\boldsymbol{X}_{n}^{m+1}}{\varphi(\Delta t)}+(1-\sigma) \boldsymbol{Y}_{\mathbf{5}} \frac{\boldsymbol{X}_{n+1}^{m+1}-\boldsymbol{X}_{n-1}^{m+1}}{2 \phi(h)}+(1-\sigma) \boldsymbol{Y}_{\mathbf{6}} \boldsymbol{X}_{n}^{m+1} \\
=\sigma \boldsymbol{Y}_{\mathbf{2}} \frac{(\phi(h))^{-\alpha_{n+1}^{m}}}{2 \cos \left(\frac{\pi \alpha_{n}^{m}}{2}\right)}\left(\sum_{j=0}^{n+1} \widehat{g}_{n, j}^{\alpha_{n+1}^{m}} \boldsymbol{X}_{j}^{m}\right) \\
+\sigma \boldsymbol{Y}_{\mathbf{3}} \frac{(\phi(h))^{-\alpha_{n+1}^{m}-1}}{2 \cos \left(\frac{\pi \alpha_{n}^{m}-1}{2}\right)}\left(\sum_{j=0}^{n+1} \widehat{g}_{n, j}^{\alpha_{n+1}^{m}-1} \boldsymbol{X}_{j}^{m}\right) \\
+\boldsymbol{Y}_{\mathbf{4}} \frac{\boldsymbol{X}_{n}^{m}}{\varphi(\triangle t)}-\sigma \boldsymbol{Y}_{\mathbf{5}} \frac{\boldsymbol{X}_{n+1}^{m}-\boldsymbol{X}_{n-1}^{m}}{2 \phi(h)}-\sigma \boldsymbol{Y}_{\mathbf{6}} \boldsymbol{X}_{n}^{m}-\sum_{j=1}^{m-1} \bar{w}_{j}^{\mu_{n}^{m}} \boldsymbol{X}_{n}^{m-j}
\end{aligned}
$$

where

$$
\bar{w}_{j}=w_{j+1}-w_{j}, \quad j=1,2, \ldots, m-2, \quad \bar{w}_{m-1}=-w_{m-1},
$$

and

$$
w_{j}=\left[(j+1)^{2-\mu}-j^{2-\mu}\right], \quad j=1,2, \ldots, m-1 .
$$

and

$$
\widehat{g}_{n, j}^{\alpha_{n}^{m}}= \begin{cases}g_{n-j+1}^{\alpha_{n}^{m}} & \text { if } j<n-1, \\ g_{0}^{\alpha_{n}^{m}}+g_{2}^{\alpha_{n}^{m}} & \text { if } j=n-1, \\ 2 g_{1}^{\alpha_{n}^{m}} & \text { if } j=n, \\ g_{0}^{\alpha_{n}^{m}}+g_{2}^{\alpha_{n}^{m}} & \text { if } j=n+1, \\ g_{j-n+1}^{\alpha_{n}^{m}} & \text { if } j>n+1 .\end{cases}
$$


After some simplification we have:

$$
\begin{aligned}
& \left(-\left(g_{0}^{\alpha_{n+1}^{m+1}}+g_{2}^{\alpha_{n+1}^{m+1}}\right)(1-\sigma) \boldsymbol{Y}_{\mathbf{2}} \frac{(\phi(h))^{-\alpha_{n+1}^{m+1}}}{2 \cos \left(\frac{\pi \alpha_{n}^{m}}{2}\right)}\right. \\
& \left.-\left(g_{0}^{\alpha_{n+1}^{m+1}-1}+g_{2}^{\alpha_{n+1}^{m+1}-1}\right)(1-\sigma) \boldsymbol{Y}_{\mathbf{3}} \frac{(\phi(h))^{-\alpha_{n+1}^{m+1}-1}}{2 \cos \left(\frac{\pi \alpha_{n}^{m}-1}{2}\right)}+(1-\sigma) \boldsymbol{Y}_{\mathbf{5}} \frac{1}{2 \phi(h)}\right) \boldsymbol{X}_{n+1}^{m+1} \\
& +\left(\boldsymbol{Y}_{\mathbf{1}} \frac{(\Delta t)^{2-\mu_{n}^{m}}}{2 \Gamma\left(3-\mu_{n}^{m}\right)(\varphi(\Delta t))}-2 g_{1}^{\alpha_{n+1}^{m+1}}(1-\sigma) \boldsymbol{Y}_{\mathbf{2}} \frac{(\phi(h))^{-\alpha_{n+1}^{m+1}}}{2 \cos \left(\frac{\pi \alpha_{n}^{m}}{2}\right)}\right. \\
& \left.-2 g_{1}^{\alpha_{n+1}^{m+1}-1}(1-\sigma) \boldsymbol{Y}_{\mathbf{3}} \frac{(\phi(h))^{-\alpha_{n+1}^{m+1}-1}}{2 \cos \left(\frac{\pi \alpha_{n}^{m}-1}{2}\right)}+\boldsymbol{Y}_{\mathbf{4}} \frac{1}{\varphi(\Delta t)}+(1-\sigma) \boldsymbol{Y}_{\mathbf{6}}\right) \boldsymbol{X}_{n}^{m+1} \\
& +\left(-\left(g_{0}^{\alpha_{n+1}^{m+1}}+g_{2}^{\alpha_{n+1}^{m+1}}\right)(1-\sigma) \boldsymbol{Y}_{\mathbf{2}} \frac{(\phi(h))^{-\alpha_{n+1}^{m+1}}}{2 \cos \left(\frac{\pi \alpha_{n}^{m}}{2}\right)}\right. \\
& \left.-\left(g_{0}^{\alpha_{n+1}^{m+1}-1}+g_{2}^{\alpha_{n+1}^{m+1}-1}\right)(1-\sigma) \boldsymbol{Y}_{\mathbf{3}} \frac{(\phi(h))^{-\alpha_{n+1}^{m+1}-1}}{2 \cos \left(\frac{\pi \alpha_{n}^{m}-1}{2}\right)}-(1-\sigma) \boldsymbol{Y}_{\mathbf{5}} \frac{1}{2 \phi(h)}\right) \boldsymbol{X}_{n-1}^{m+1} \\
& -(1-\sigma) \boldsymbol{Y}_{\mathbf{2}} \frac{(\phi(h))^{-\alpha_{n+1}^{m+1}}}{2 \cos \left(\frac{\pi \alpha_{n}^{m}}{2}\right)}\left(\sum_{j=0, j \neq\{n-1, n, n+1\}}^{N} \widehat{g}_{n, j}^{\alpha_{n+1}^{m+1}} \boldsymbol{X}_{j}^{m+1}\right) \\
& -(1-\sigma) \boldsymbol{Y}_{\mathbf{3}} \frac{(\phi(h))^{-\alpha_{n+1}^{m+1}-1}}{2 \cos \left(\frac{\pi \alpha_{n}^{m}-1}{2}\right)}\left(\sum_{j=0, j \neq\{n-1, n, n+1\}}^{N} \widehat{g}_{n, j}^{\alpha_{n+1}^{m+1}-1} \boldsymbol{X}_{j}^{m+1}\right) \\
& =\left(\left(g_{0}^{\alpha_{n+1}^{m}}+g_{2}^{\alpha_{n+1}^{m}}\right) \sigma \boldsymbol{Y}_{\mathbf{2}} \frac{(\phi(h))^{-\alpha_{n+1}^{m}}}{2 \cos \left(\frac{\pi \alpha_{n}^{m}}{2}\right)}\right. \\
& \left.+\left(g_{0}^{\alpha_{n+1}^{m}-1}+g_{2}^{\alpha_{n+1}^{m}-1}\right) \sigma \boldsymbol{Y}_{\mathbf{3}} \frac{(\phi(h))^{-\alpha_{n+1}^{m}-1}}{2 \cos \left(\frac{\pi \alpha_{n}^{m}-1}{2}\right)}-\sigma \boldsymbol{Y}_{\mathbf{5}} \frac{1}{2 \phi(h)}\right) \boldsymbol{X}_{n+1}^{m} \\
& +\left(2 g_{1}^{\alpha_{n+1}^{m+1}} \sigma \boldsymbol{Y}_{\mathbf{2}} \frac{(\phi(h))^{-\alpha_{n+1}^{m+1}}}{2 \cos \left(\frac{\pi \alpha_{n}^{m}}{2}\right)}\right. \\
& \left.+2 g_{1}^{\alpha_{n+1}^{m+1}-1} \sigma \boldsymbol{Y}_{\mathbf{3}} \frac{(\phi(h))^{-\alpha_{n+1}^{m+1}-1}}{2 \cos \left(\frac{\pi \alpha_{n}^{m}-1}{2}\right)}+\boldsymbol{Y}_{\mathbf{4}} \frac{1}{\varphi(\Delta t)}-\sigma \boldsymbol{Y}_{\mathbf{6}}\right) \boldsymbol{X}_{n}^{m} \\
& +\left(\left(g_{0}^{\alpha_{n+1}^{m}}+g_{2}^{\alpha_{n+1}^{m}}\right) \sigma \boldsymbol{Y}_{\mathbf{2}} \frac{(\phi(h))^{-\alpha_{n+1}^{m}}}{2 \cos \left(\frac{\pi \alpha_{n}^{m}}{2}\right)}\right. \\
& \left.+\left(g_{0}^{\alpha_{n+1}^{m}-1}+g_{2}^{\alpha_{n+1}^{m}-1}\right) \sigma \boldsymbol{Y}_{\mathbf{3}} \frac{(\phi(h))^{-\alpha_{n+1}^{m}-1}}{2 \cos \left(\frac{\pi \alpha_{n}^{m}-1}{2}\right)}-\sigma \boldsymbol{Y}_{\mathbf{5}} \frac{1}{2 \phi(h)}\right) \boldsymbol{X}_{n-1}^{m} \\
& +\sigma \boldsymbol{Y}_{\mathbf{2}} \frac{(\phi(h))^{-\alpha_{n+1}^{m}}}{2 \cos \left(\frac{\pi \alpha_{n}^{m}}{2}\right)}\left(\sum_{j=0, j \neq\{n-1, n, n+1\}}^{N} \widehat{g}_{n, j}^{\alpha_{n+1}^{m}} \boldsymbol{X}_{j}^{m}\right) \\
& +\sigma \boldsymbol{Y}_{\mathbf{3}} \frac{(\phi(h))^{-\alpha_{n+1}^{m}-1}}{2 \cos \left(\frac{\pi \alpha_{n}^{m}-1}{2}\right)}\left(\sum_{j=0, j \neq\{n-1, n, n+1\}}^{N} \widehat{g}_{n, j}^{\alpha_{n+1}^{m}-1} \boldsymbol{X}_{j}^{m}\right) \\
& -\sum_{j=1}^{m-1} \bar{w}_{j}^{\mu_{n}^{m}} \boldsymbol{X}_{n}^{m-j}
\end{aligned}
$$


this scheme takes the following composite form:

$$
\begin{aligned}
\boldsymbol{A}_{1} \boldsymbol{X}_{n+1}^{m+1}+\boldsymbol{A}_{2} \boldsymbol{X}_{n}^{m+1}+\boldsymbol{A}_{3} \boldsymbol{X}_{n-1}^{m+1}-(1-\sigma) \sum_{j=0, j \neq\{n-1, n, n+1\}}^{N}\left(\boldsymbol{A}_{4, j}+\boldsymbol{A}_{5, j}\right) \boldsymbol{X}_{j}^{m+1} \\
=\boldsymbol{B}_{1} \boldsymbol{X}_{n+1}^{m}+\boldsymbol{B}_{2} \boldsymbol{X}_{n}^{m}+\boldsymbol{B}_{3} \boldsymbol{X}_{n-1}^{m}+\sigma \sum_{j=0, j \neq\{n-1, n, n+1\}}^{N}\left(\boldsymbol{B}_{4, j}+\boldsymbol{B}_{5, j}\right) \boldsymbol{X}_{j}^{m} \\
\quad-\sum_{j=1}^{m-1} \bar{w}_{j}^{\mu_{n}^{m}} \boldsymbol{X}_{n}^{m-j} .
\end{aligned}
$$

Depending on the von Neumann stability method, we presume

$$
\boldsymbol{X}_{n}^{m}=\boldsymbol{\xi}^{m} \boldsymbol{\Upsilon} e^{i n \beta h}
$$

such that $\beta \in \mathbb{R}, i=\sqrt{-1}, \boldsymbol{\Upsilon} \in \mathbb{R}^{4 \times 1}$, and $\xi \in \mathbb{R}^{4 \times 4}$ is an amplification element. Putting Eq. (28) into Eq. (27), after some manipulation we obtain the following form of the amplification matrix:

$$
\begin{aligned}
\boldsymbol{\xi}= & {\left[\boldsymbol{A}_{1} e^{i \beta h}+\boldsymbol{A}_{2}+\boldsymbol{A}_{3} e^{-i \beta h}-(1-\sigma) \sum_{j=0, j \neq\{n-1, n, n+1\}}^{N}\left(\boldsymbol{A}_{4, j}+\boldsymbol{A}_{5, j}\right) e^{i(j-n) \beta h}\right]^{-1} } \\
& \times\left[\boldsymbol{B}_{1} e^{i \beta h}+\boldsymbol{B}_{2}+\boldsymbol{B}_{3} e^{-i \beta h}+\sigma \sum_{j=0, j \neq\{n-1, n, n+1\}}^{N}\left(\boldsymbol{B}_{4, j}+\boldsymbol{B}_{5, j}\right) e^{i(j-n) \beta h}+\sum_{1}^{m-1} \bar{w}_{j}^{\mu_{n}^{m}} \xi^{-j}\right] .
\end{aligned}
$$

The scheme will be stable when $\|\xi\| \leq 1$. As in $[50,51,66]$, we consider on the right side the time-independent $\xi=I$ so:

$$
\sum_{1}^{m-1} \bar{w}_{j}^{\mu_{n}^{m}} \boldsymbol{\xi}^{-j}=\left(2^{2-\mu_{n}^{m}}-1\right) \boldsymbol{I},
$$

therefore,

$$
\begin{aligned}
\boldsymbol{\xi}= & {\left[\boldsymbol{A}_{1} e^{i \beta h}+\boldsymbol{A}_{2}+\boldsymbol{A}_{3} e^{-i \beta h}-(1-\sigma) \sum_{j=0, j \neq\{n-1, n, n+1\}}^{N}\left(\boldsymbol{A}_{4, j}+\boldsymbol{A}_{5, j}\right) e^{i(j-n) \beta h}\right]^{-1} } \\
& \times\left[\boldsymbol{B}_{1} e^{i \beta h}+\boldsymbol{B}_{2}+\boldsymbol{B}_{3} e^{-i \beta h}+\sigma \sum_{j=0, j \neq\{n-1, n, n+1\}}^{N}\left(\boldsymbol{B}_{4, j}+\boldsymbol{B}_{5, j}\right) e^{i(j-n) \beta h}+\left(2^{2-\mu_{n}^{m}}-1\right) \boldsymbol{I}\right] .
\end{aligned}
$$

Here $\xi$ depends on $\mu_{n}^{m}, \sigma, h$, and $\Delta t$, as we see from Eq. (30).

Scheme (27) will be stable as soon as the following relation is satisfied:

$$
\begin{gathered}
\|\left[\boldsymbol{A}_{1} e^{i \beta h}+\boldsymbol{A}_{2}+\boldsymbol{A}_{3} e^{-i \beta h}-(1-\sigma) \sum_{j=0, j \neq\{n-1, n, n+1\}}^{N}\left(\boldsymbol{A}_{4, j}+\boldsymbol{A}_{5, j}\right) e^{i(j-n) \beta h}\right]^{-1} \\
\times\left[\boldsymbol{B}_{1} e^{i \beta h}+\boldsymbol{B}_{2}+\boldsymbol{B}_{3} e^{-i \beta h}+\sigma \sum_{j=0, j \neq\{n-1, n, n+1\}}^{N}\left(\boldsymbol{B}_{4, j}+\boldsymbol{B}_{5, j}\right) e^{i(j-n) \beta h}+\left(2^{2-\mu_{n}^{m}}-1\right) \boldsymbol{I}\right] \|
\end{gathered}
$$$$
\leq 1
$$ 


\subsection{Truncation error}

Depending on definition of truncation error (15), (18), and (12), and from relations (11a)(11f), we have

$$
T_{n}^{m}=O\left((\phi(h))^{2}+(\varphi(\Delta t))^{2}\right),
$$

but $\phi(h)=h+O(h)^{2}$ and $\varphi(\Delta t)=\Delta t+O(\Delta t)^{2}$, so

$$
T_{n}^{m}=O\left(h^{2}+(\triangle t)^{2}\right)
$$

Remark 4.1 It is well known that the Lax-Richtmyer equivalence theorem says: for consistent numerical approximations, stability and convergence are equivalent in the integer order differential equations. Yuste and Murillo in 2012 generalized this theorem to the fractional derivative case. They proved in [67, Sect. 4] that for a whole class of the fractional difference algorithms the consistency and stability of the difference scheme implies its convergence. Therefore our scheme is convergent under condition (31).

\section{Numerical simulations}

In the present section, three test examples are introduced to show the application of the used technique to solve the proposed nonlinear coupled model of a time-space fractional variable-order hyperbolic and parabolic equations. To check the accuracy of the proposed scheme, we estimate the $L_{\infty}$-error (when $\mu(x, t)=\alpha(x, t)=2$, the derivatives of integer order) using formulas

$$
E_{1}=\max \left|u_{\text {exact }}\left(x_{n}, t_{m}\right)-u_{\text {approx }}\left(x_{n}, t_{m}\right)\right|, \quad E_{2}=\max \left|\theta_{\text {exact }}\left(x_{n}, t_{m}\right)-\theta_{\text {approx }}\left(x_{n}, t_{m}\right)\right|
$$

for all $n, m$. Let $\varphi(\Delta t)=1-e^{-\Delta t}$ and $\phi(h)=\sinh (\Delta x)$.

Example 1 ([1]) We deal with the system (10) for:

$$
\begin{aligned}
f(x, t)= & e^{-x} \cos t+\left(\beta_{1}-1\right) e^{-x}(1-\cos t) \\
& +2 \gamma e^{-2 x}(1-\cos t)^{2}-3 \delta e^{-3 x}(1-\cos t)^{3}, \\
l(x, t)= & (1+a) e^{-x} \sin t-\left(1+b e^{-x} \sin t\right) e^{-x}(1-\cos t)+2 \alpha^{*} e^{-2 x}(1-\cos t)^{2},
\end{aligned}
$$

and the exact solutions when $\mu(x, t)=\alpha(x, t)=2$ (derivatives of integer order) are

$$
u(x, t)=e^{-x}(1-\cos t)=\theta(x, t), \quad 0 \leq x \leq 1 .
$$

The boundary conditions are

$$
u(0, t)=1-\cos t, \quad u(1, t)=\frac{1-\cos t}{e}, \quad \theta(0, t)=1-\cos t, \quad \theta(1, t)=\frac{1-\cos t}{e},
$$

and the initial conditions are

$$
u(x, 0)=0, \quad u_{t}(x, 0)=0, \quad \theta(x, 0)=0,
$$

and $a=0.1, b=0.1, \alpha^{*}=1, \beta_{1}=0.5, \beta_{2}=0.5, \gamma=1, \delta=0.8$. 
Table 1 Comparison of $E_{1}$ and $E_{2}$, the maximum absolute errors, for different values of $N, M$ in Example 1, when $0<t \leq 1$

\begin{tabular}{|c|c|c|c|c|c|c|c|}
\hline \multirow[t]{2}{*}{ N } & \multirow[t]{2}{*}{$M$} & \multicolumn{3}{|l|}{$E_{1}$} & \multicolumn{3}{|l|}{$E_{2}$} \\
\hline & & $\overline{\sigma=1}$ & $\sigma=0.5$ & $\sigma=0$ & $\overline{\sigma=1}$ & $\sigma=0.5$ & $\sigma=0$ \\
\hline 10 & 10 & $3.5478 \mathrm{e}-01$ & $1.1329 \mathrm{e}-02$ & $4.4571 \mathrm{e}-03$ & $4.3610 \mathrm{e}-01$ & $5.8361 \mathrm{e}-02$ & $2.2423 e-02$ \\
\hline 25 & 25 & $4.1842 \mathrm{e}+00$ & $4.6783 \mathrm{e}-03$ & $1.9563 \mathrm{e}-03$ & divergent & $4.3784 \mathrm{e}-03$ & $5.6345 \mathrm{e}-03$ \\
\hline 40 & 40 & $9.8712 e+00$ & $7.7532 \mathrm{e}-04$ & $8.3712 \mathrm{e}-04$ & divergent & $1.3741 \mathrm{e}-03$ & $1.1524 \mathrm{e}-03$ \\
\hline 50 & 50 & divergent & $1.0763 e-04$ & $4.5137 \mathrm{e}-04$ & divergent & $7.4723 e-04$ & $7.7859 \mathrm{e}-04$ \\
\hline
\end{tabular}

Table 2 Comparison of $E_{1}$ and $E_{2}$, the maximum absolute errors, for different values of $N, M$ in Example 1, when $0<t \leq 5$

\begin{tabular}{|c|c|c|c|c|c|c|c|}
\hline \multirow[t]{2}{*}{ N } & \multirow[t]{2}{*}{$M$} & \multicolumn{3}{|l|}{$E_{1}$} & \multicolumn{3}{|l|}{$E_{2}$} \\
\hline & & $\overline{\sigma=1}$ & $\sigma=0.5$ & $\sigma=0$ & $\overline{\sigma=1}$ & $\sigma=0.5$ & $\sigma=0$ \\
\hline 40 & 40 & divergent & $3.4226 \mathrm{e}-01$ & $2.1399 \mathrm{e}-01$ & divergent & $8.0341 \mathrm{e}-01$ & $1.8633 \mathrm{e}-01$ \\
\hline 60 & 60 & divergent & $6.8113 e-02$ & $8.3989 \mathrm{e}-02$ & divergent & $5.65394 \mathrm{e}-02$ & $2.9847 \mathrm{e}-02$ \\
\hline 60 & 80 & divergent & $9.9546 \mathrm{e}-03$ & $1.0302 \mathrm{e}-02$ & divergent & 7.4853e-03 & $1.0301 \mathrm{e}-02$ \\
\hline
\end{tabular}

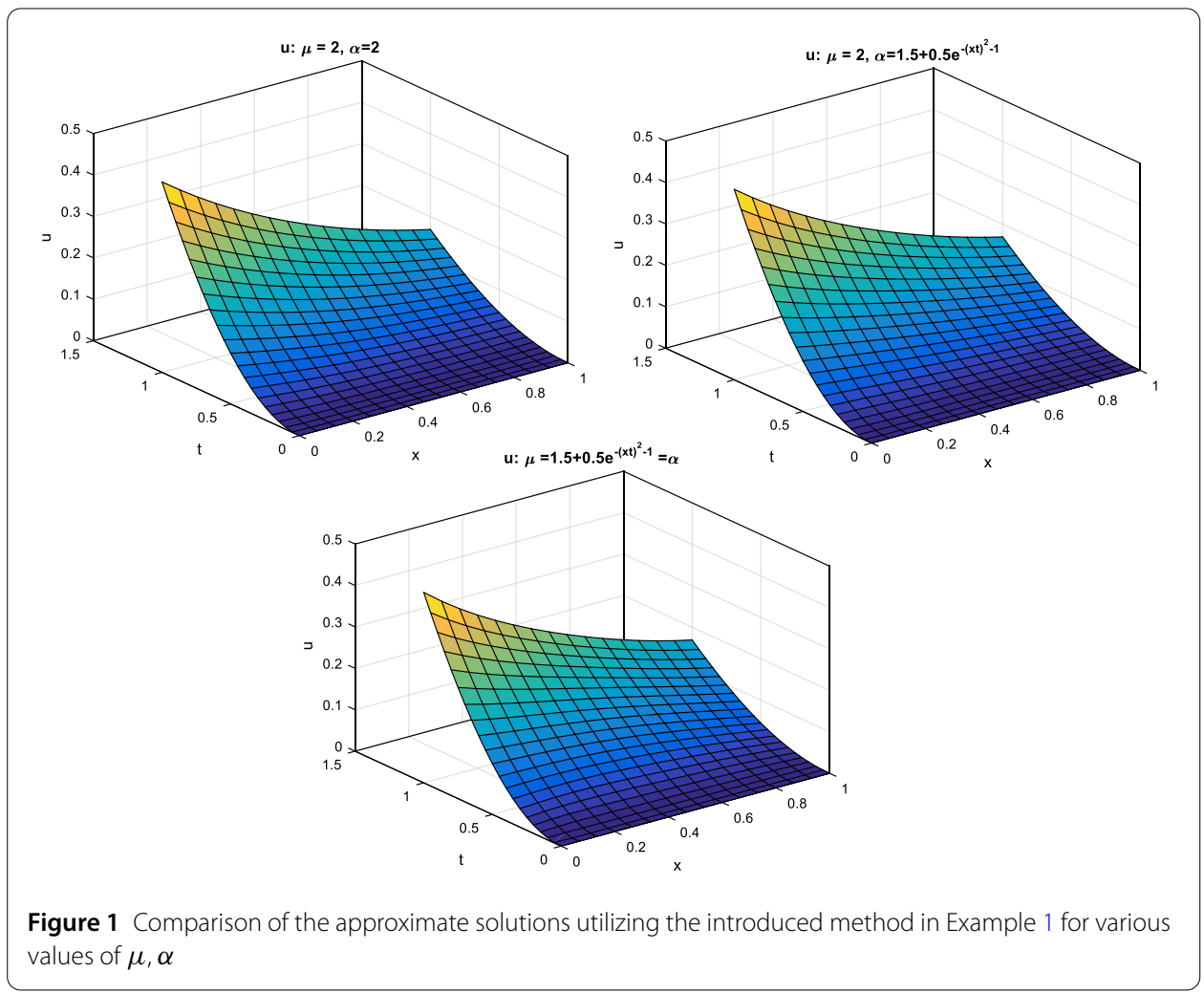

From Table 1 we see that $E_{1}$ and $E_{2}$ are reduced when $N$ and $M$ grow with $\sigma=0,0.5$ and $0<t \leq 1$ and deduce that the approximations obtained by the explicit NSFDM do not converge because the condition of stability is not satisfied in this case.

Also, when $0<t \leq 5$, we deduce the same conclusion from Table 2 .

In Fig. 1 we see the approximation of $u$ which was achieved by the introduced WANSFD scheme for various values of $\alpha, \mu$ where $\sigma=0.5$ and $0<t \leq 1$.

In Fig. 2 we see the approximation of $u$ which was achieved by the introduced WANSFD scheme for various values of $\alpha, \mu$ where $\sigma=0.5$ and $0<t \leq 5$. 


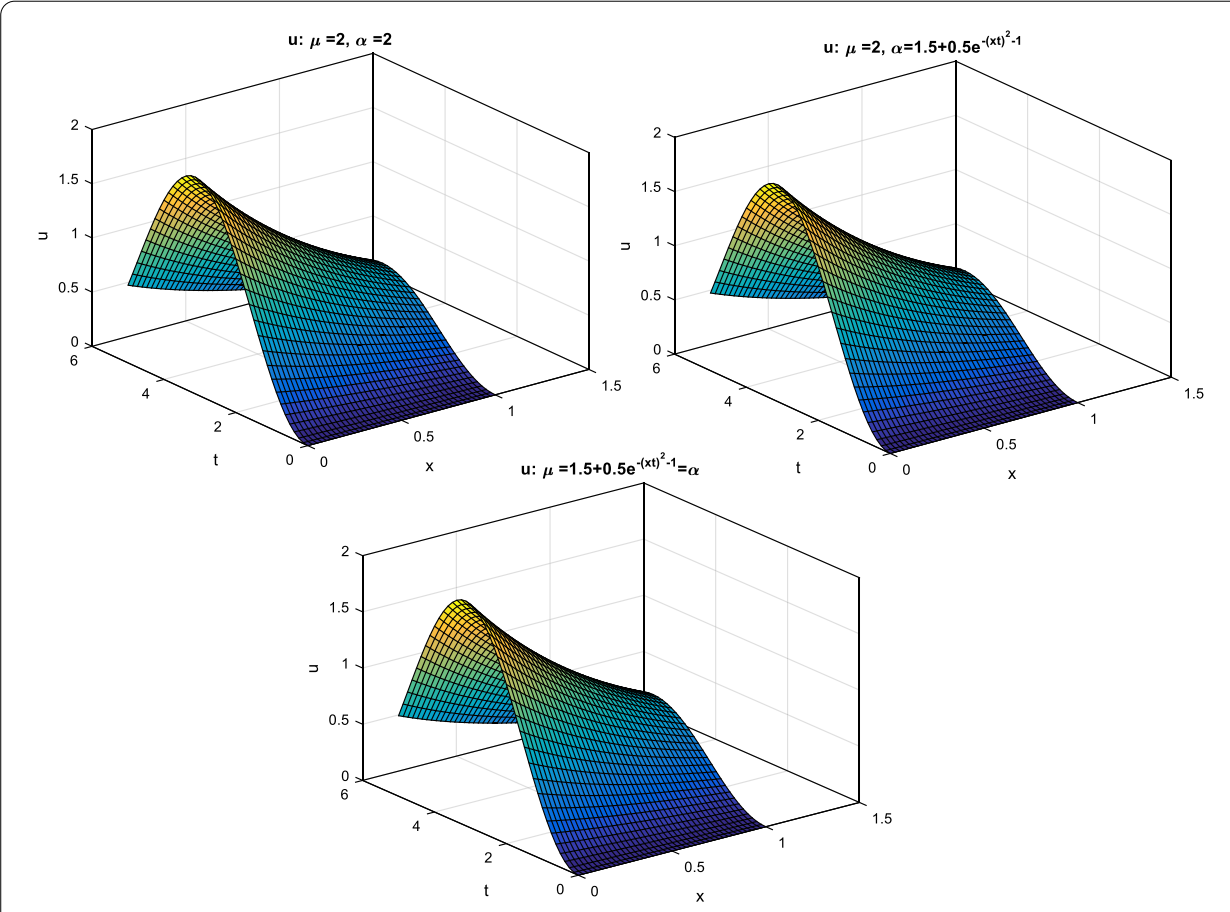

Figure 2 Comparison of the approximate solutions utilizing the introduced method in Example 1 for various values of $\mu, \alpha$

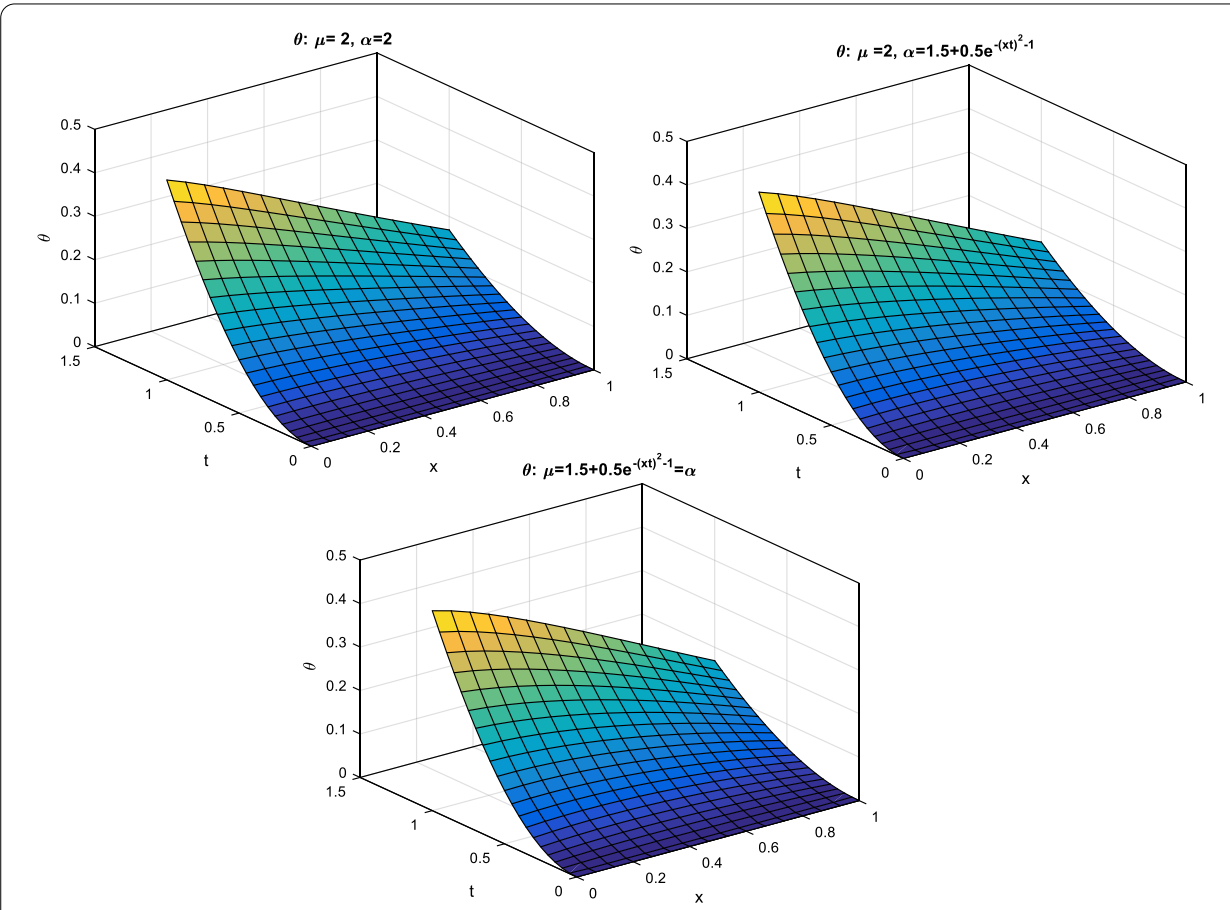

Figure 3 Comparison of the numerical solutions using the introduced method in Example 1 for different values of $\mu, \alpha$ 
In Fig. 3 we see the approximation of $\theta$ which was achieved by the introduced WANSFD scheme for various values of $\alpha, \mu$ where $\sigma=0.5$.

Example 2 ([55]) We treat system (14) for

$$
f(x, t)=2 e^{-t} \sin x-e^{-3 t} \cos ^{2} x \sin x, \quad l(x, t)=-e^{-3 t} \cos ^{3} x,
$$

and the analytic exact solutions for $\mu=2, \alpha=2$ (integer order derivative) are given by

$$
u(x, t)=e^{-t} \sin x, \quad \theta(x, t)=e^{-t} \cos x, \quad 0 \leq x \leq \pi
$$

The boundary conditions are

$$
u(0, t)=0, \quad u(\pi, t)=0, \quad \theta(0, t)=e^{-t}, \quad \theta(\pi, t)=-e^{-t},
$$

and the initial conditions are

$$
u(x, 0)=\sin x, \quad u_{t}(x, 0)=-\sin x, \quad \theta(x, 0)=\cos x .
$$

From Table 3 we see that $E_{1}$ and $E_{2}$ are reduced when $N$ and $M$ grow when $\sigma=0,0.5$, $0<t \leq 1$ and deduce that the numerical approximation utilizing the explicit NSFDM is not convergent because the condition of stability is not satisfied in this case.

In Fig. 4 we see the approximation of $u$ which was achieved by the introduced WANSFD scheme for various values of $\mu, \alpha$ where $\sigma=0.5$.

In Fig. 5 we see the approximation of $\theta$ which was achieved by the introduced WANSFD scheme for various values of $\mu, \alpha$ where $\sigma=0.5$.

Figure 6 illustrates the behavior of the approximations of $u$ obtained by the WANSFD technique when $(\sigma=0.5)$ at $t=0.5$, for various values of $\mu$ and $\alpha=2$.

Figure 7 illustrates the behavior of the numerical approximations of $u$ obtained by the WANSFD technique when $(\sigma=0.5)$ at $x=0.7$, for various values of $\alpha$ and $\mu=2$.

Figure 8 illustrates the behavior of the numerical approximations of $\theta$ obtained by the WANSFD method when $(\sigma=0.5)$ at $t=0.5$, for various values of $\mu$ and $\alpha=2$.

Figure 9 illustrates the behavior of the numerical approximations of $\theta$ obtained by the

\begin{tabular}{|c|c|c|c|c|c|c|c|}
\hline \multirow[t]{2}{*}{ N } & \multirow[t]{2}{*}{$M$} & \multicolumn{3}{|l|}{$E_{1}$} & \multicolumn{3}{|l|}{$E_{2}$} \\
\hline & & $\sigma=1$ & $\sigma=0.5$ & $\sigma=0$ & $\sigma=1$ & $\sigma=0.5$ & $\sigma=0$ \\
\hline 10 & 10 & $5.2998 \mathrm{e}-00$ & $4.5278 \mathrm{e}-02$ & $2.0864 \mathrm{e}-02$ & $6.2518 \mathrm{e}-01$ & $2.0721 \mathrm{e}-02$ & $2.0668 \mathrm{e}-02$ \\
\hline 25 & 25 & divergent & $6.4189 e-03$ & $8.9760 \mathrm{e}-03$ & divergent & $9.6235 e-03$ & $8.9655 e-03$ \\
\hline 40 & 40 & divergent & $3.7251 \mathrm{e}-03$ & $2.5102 \mathrm{e}-03$ & divergent & $5.1112 \mathrm{e}-03$ & $3.8879 e-03$ \\
\hline 50 & 50 & divergent & $1.0267 \mathrm{e}-03$ & $9.5183 e-04$ & divergent & $8.9926 \mathrm{e}-04$ & $7.1533 e-04$ \\
\hline
\end{tabular}
WANSFD method when $(\sigma=0.5)$ at $x=0.7$, for various values of $\alpha$ and $\mu=2$.

Table 3 Comparison of $E_{1}$ and $E_{2}$, the maximum absolute errors, for different values of $N, M$ in Example 2 


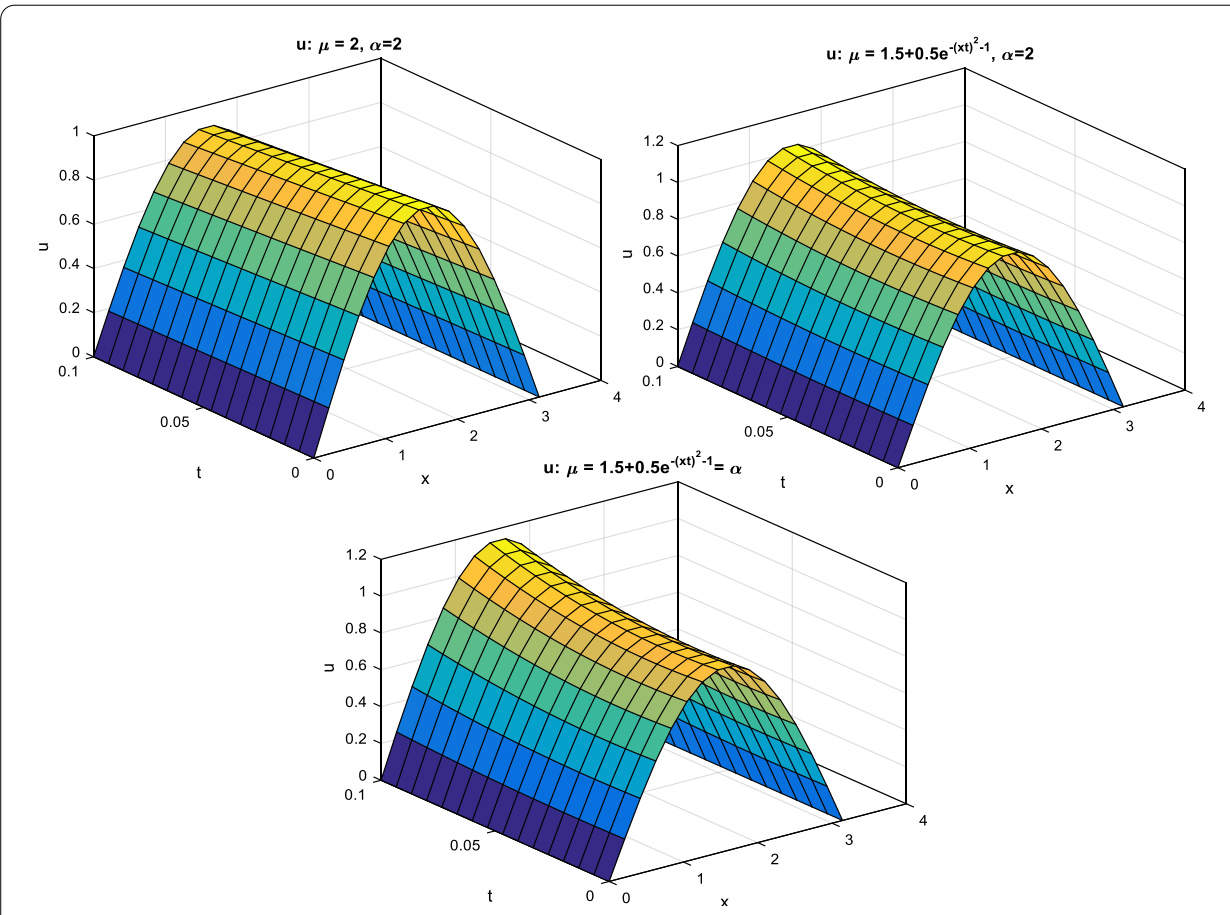

Figure 4 Comparison of the approximate solutions utilizing the introduced method in Example 2 for different values of $\mu, \alpha$

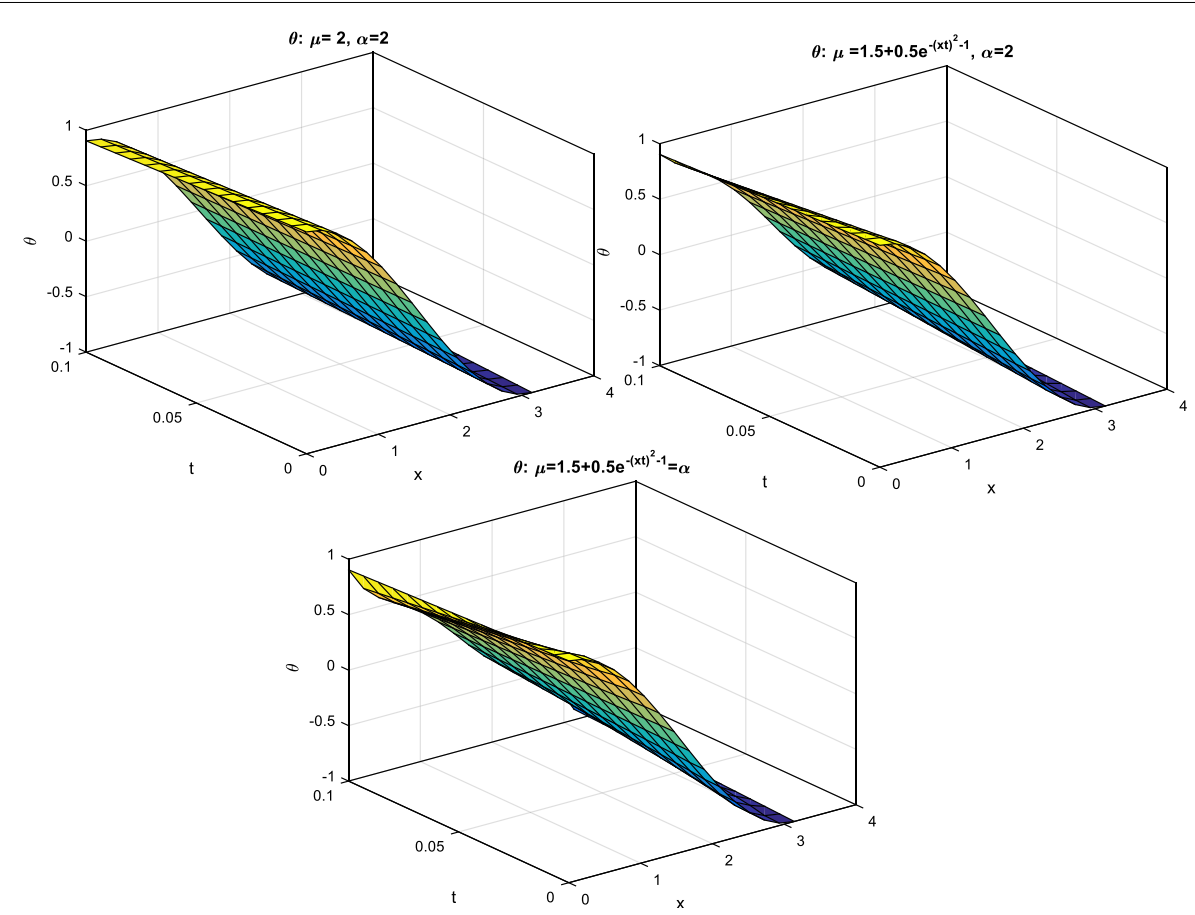

Figure 5 Comparison of the numerical solutions using the introduced method in Example 2 for different values of $\mu, \alpha$ 
Figure 6 Behavior of the solutions using the introduced schemes in Example 2 for various values of $\mu$ when $N=M=10$ at $t=0.5$

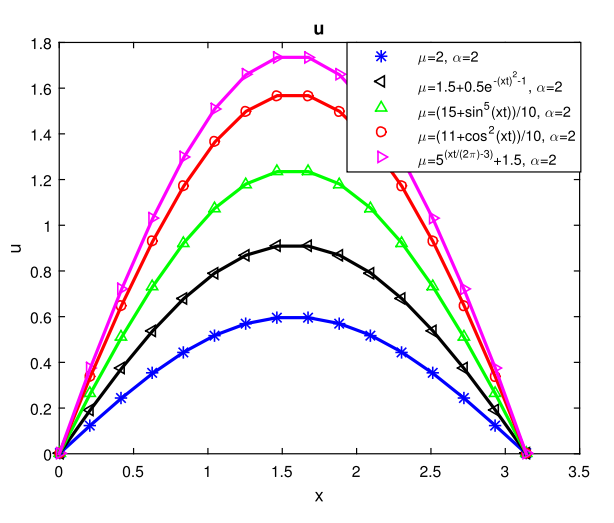

Figure 7 Behavior of the solutions using the introduced schemes in Example 2 for various values of $\mu$ when $N=M=16$ at $x=0.7$

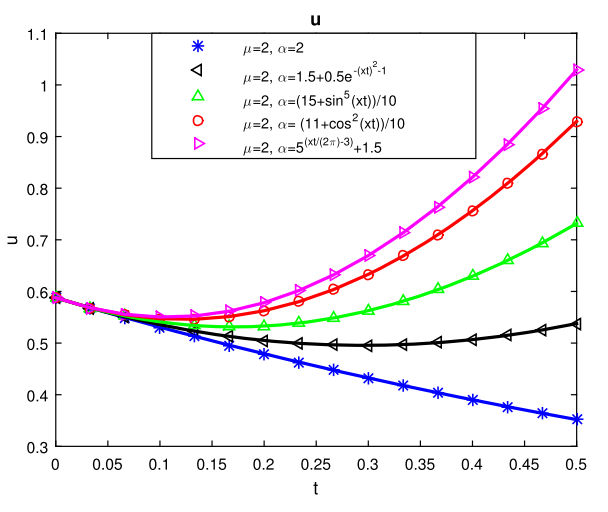

Figure 8 Behavior of the solutions using the introduced schemes in Example 2 for various values of $\mu$ when $N=M=16$ at $t=0.5$

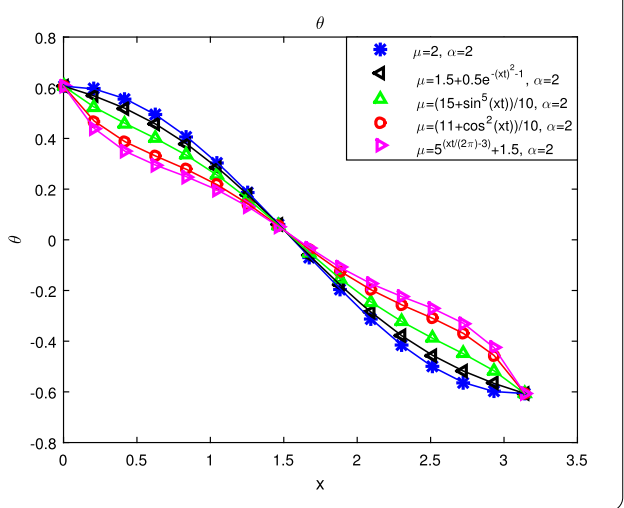

Figure 9 Behavior of the solutions using the introduced schemes in Example 2 for different values of $\mu$ when $N=M=16$ at $x=0.7$

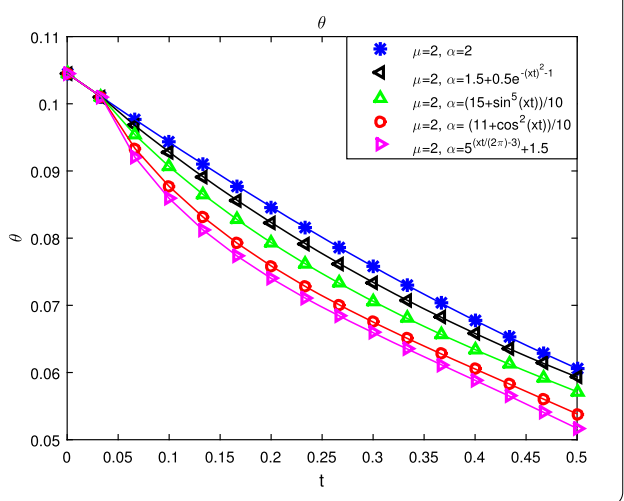


Example 3 ([12]) Let us consider system (17) where

$$
\begin{aligned}
f(x, t)= & \frac{2}{1+x^{2}}-\frac{2\left(1+t^{2}\right)\left(3 x^{2}-1\right)}{\left(1+x^{2}\right)^{3}}\left(2+\frac{2 x\left(1+t^{2}\right)}{\left(1+x^{2}\right)^{2}} \frac{1+t}{1+x^{2}}\right) \\
& +\frac{2 x(1+t)}{\left(1+x^{2}\right)^{2}}\left(2-\frac{2 x\left(1+t^{2}\right)}{\left(1+x^{2}\right)^{2}} \frac{1+t}{1+x^{2}}\right), \\
l(x, t)= & \frac{1}{1+x^{2}}-\frac{4 x t}{\left(1+x^{2}\right)^{2}}\left(2+\frac{2 x\left(1+t^{2}\right)}{\left(1+x^{2}\right)^{2}} \frac{1+t}{1+x^{2}}\right) \\
& -\frac{2\left(3 x^{2}-1\right)(1+t)}{\left(1+x^{2}\right)^{2}} \frac{1+t}{1+x^{2}},
\end{aligned}
$$

also the analytic exact solutions for $\mu=2, \alpha=2$ (integer order derivative) are

$$
u(x, t)=\frac{1+t^{2}}{1+x^{2}}, \quad \theta(x, t)=\frac{1+t}{1+x^{2}}, \quad 0 \leq x \leq 1 .
$$

The boundary conditions are

$$
u(0, t)=1+t^{2}, \quad u(1, t)=\frac{1+t^{2}}{2}, \quad \theta(0, t)=1+t, \quad \theta(\pi, t)=\frac{1+t}{2}
$$

and the initial conditions are

$$
u(x, 0)=\frac{1}{1+x^{2}}, \quad u_{t}(x, 0)=0, \quad \theta(x, 0)=\frac{1}{1+x^{2}}
$$

From Table 4 we see that $E_{1}$ and $E_{2}$ are reduced when $N$ and $M$ grow for $\sigma=0,0.5$, $0<t \leq 1$ and deduce that the numerical approximation depending on the explicit NSFDM is not convergent because the condition of stability is not satisfied in this case.

In Fig. 10 we see the approximation of $u$ which was achieved by the introduced WANSFD scheme for various values of $\alpha, \mu$ and $\sigma=0.5$.

In Fig. 11 we see the approximation of $\theta$ which was achieved by the introduced WANSFD scheme for various values of $\alpha, \mu$ and $\sigma=0.5$.

\begin{tabular}{|c|c|c|c|c|c|c|c|}
\hline \multirow[t]{2}{*}{ N } & \multirow[t]{2}{*}{$M$} & \multicolumn{3}{|l|}{$M_{1}$} & \multicolumn{3}{|l|}{$M_{2}$} \\
\hline & & $\sigma=1$ & $\sigma=0.5$ & $\sigma=0$ & $\overline{\sigma=1}$ & $\sigma=0.5$ & $\sigma=0$ \\
\hline 6 & 5 & $4.5332 e+00$ & $8.5664 \mathrm{e}-02$ & $7.7553 e-02$ & $9.8871 \mathrm{e}-01$ & $3.4288 \mathrm{e}-02$ & $5.1149 \mathrm{e}-02$ \\
\hline 12 & 10 & divergent & $3.4421 \mathrm{e}-02$ & $1.1233 \mathrm{e}-02$ & divergent & $1.0542 \mathrm{e}-02$ & $1.01783 e-02$ \\
\hline 24 & 20 & divergent & $6.7833 e-03$ & $6.4355 \mathrm{e}-02$ & divergent & $9.3441 \mathrm{e}-03$ & $8.9178 \mathrm{e}-03$ \\
\hline 36 & 30 & divergent & $1.0499 \mathrm{e}-03$ & $7.0998 \mathrm{e}-03$ & divergent & $5.7334 \mathrm{e}-03$ & $5.1807 \mathrm{e}-03$ \\
\hline
\end{tabular}

Figure 12 illustrates the behavior of the numerical approximations of $u$ obtained by the WANSFD technique when $(\sigma=0.5)$ at $x=0.3$, for various values of $\alpha$ and $\mu=2$.

Figure 13 illustrates the behavior of the numerical approximations of $\theta$ obtained by the WANSFD method when $(\sigma=0.5)$ at $x=0.3$, for different values of $\alpha$ and $\mu=2$.

Table 4 Comparison of $E_{1}$ and $E_{2}$, the maximum absolute errors, for different values of $N, M$ in Example 3 


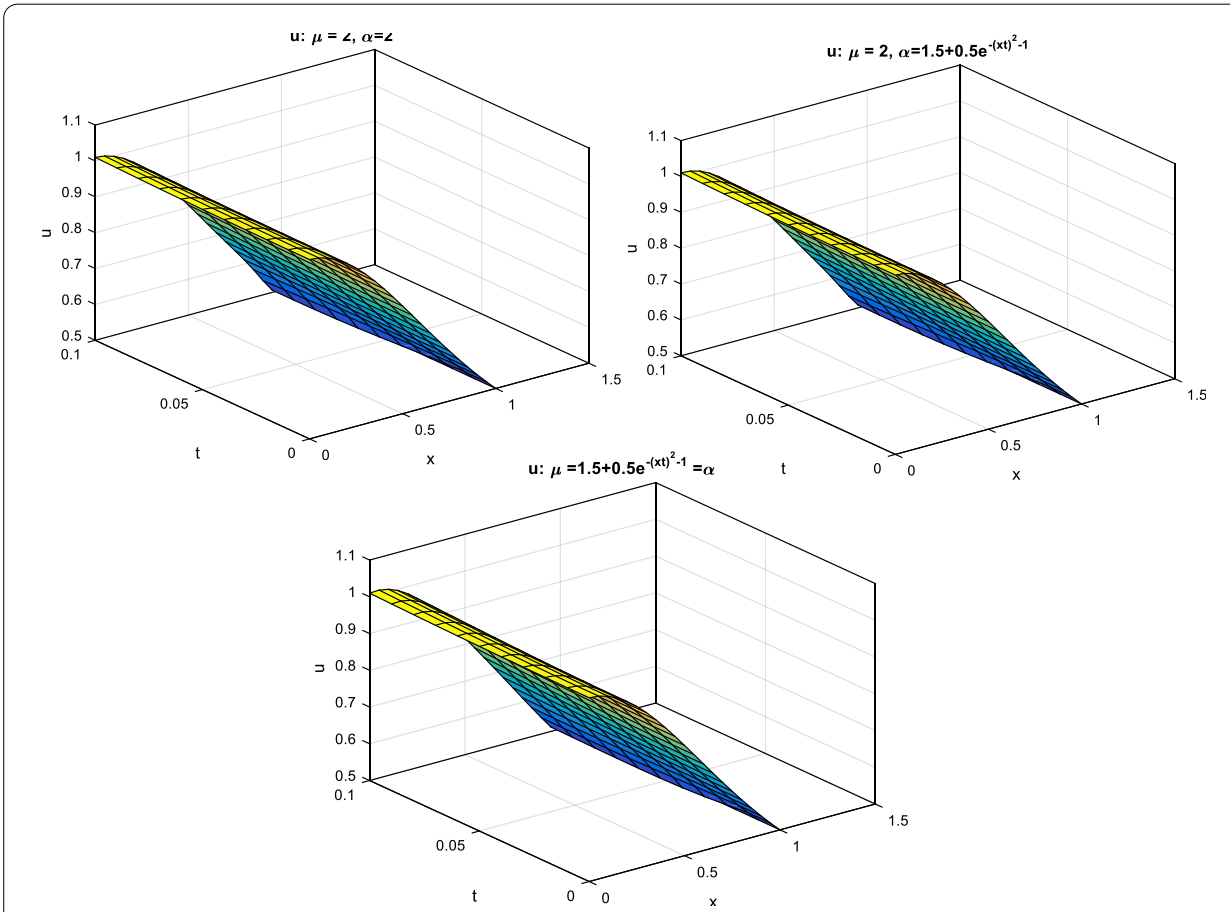

Figure 10 Comparison of the numerical solutions using the introduced method in Example 3 for various values of $\mu, \alpha$

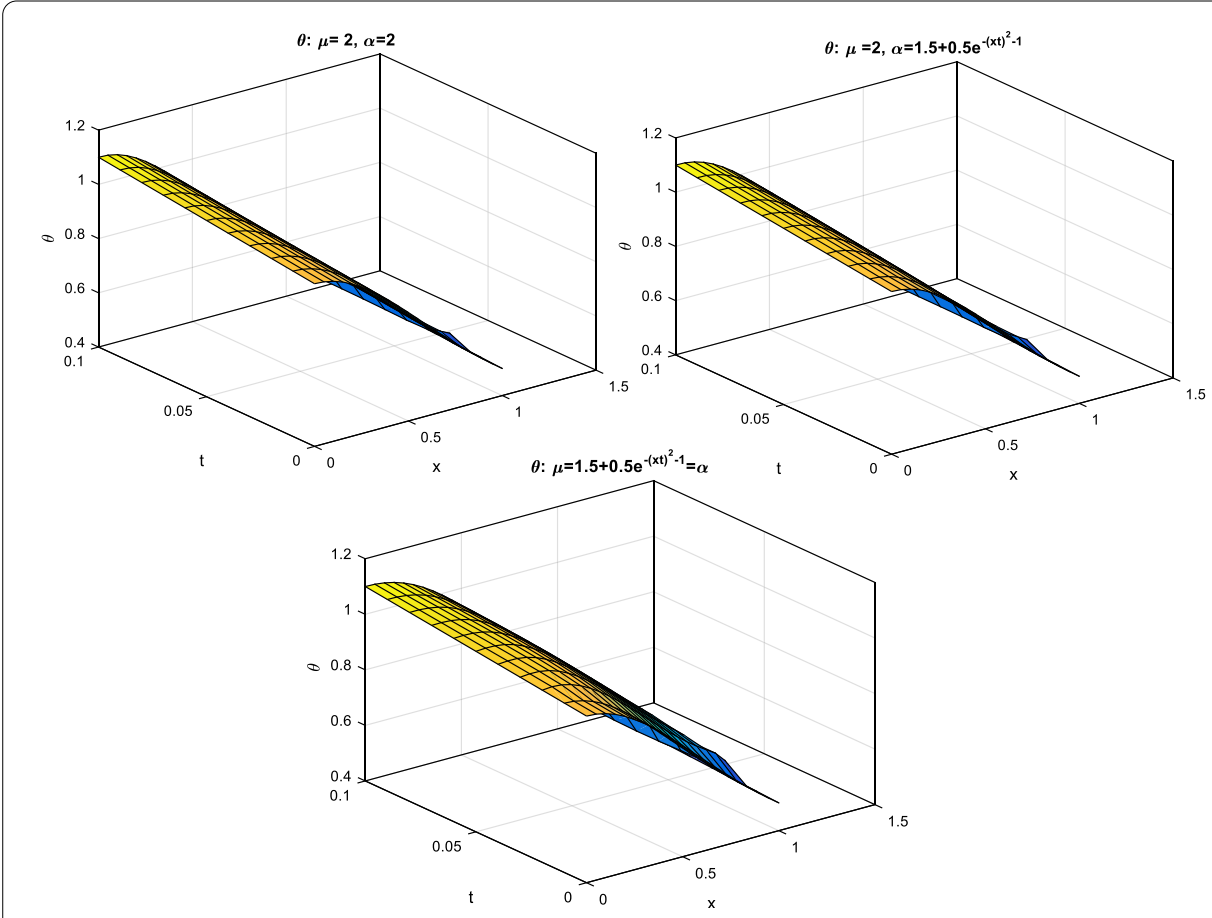

Figure 11 Comparison of the numerical solutions using the introduced method in Example 3 for various values of $\mu, \alpha$ 
Figure 12 Behavior of the solutions using the introduced schemes in Example 3 for various values of $\mu$ when $N=M=16$ at $x=0.3$

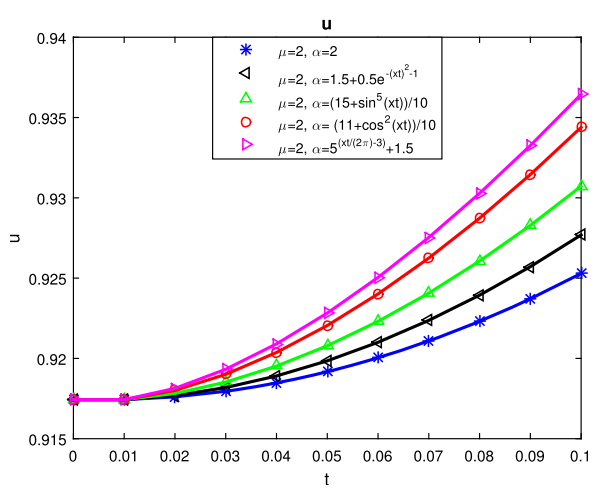

Figure 13 Behavior of the solutions using the introduced schemes in Example 3 for various values of $\mu$ when $N=M=16$ at $x=0.3$

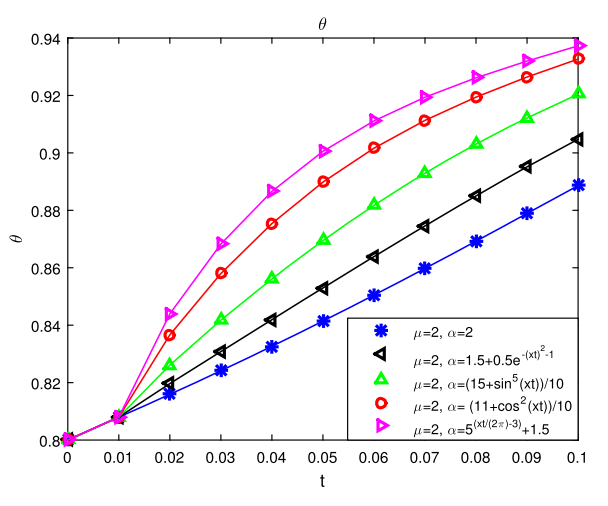

\section{Summary and conclusions}

In the paper, we introduced numerical simulations for nonlinear systems of time-space variable-order fractional, coupled hyperbolic and parabolic partial differential equations in one dimension utilizing WANSFDM. Analyzing stability of the proposed scheme is presented by a kind of John von Neumann method, also the relation which determines the truncation error is given. Some test examples are also presented. We compare the numerical results obtained by the used technique with the analytic exact solutions of the proposed model in the case of the standard derivative (integer order). We introduce many figures to show the behaviors of the solutions when $\mu$ and $\alpha$ are changed.

\section{Acknowledgements}

The author would like to thank the editor, associate editor, and the anonymous reviewers for their many helpful comments and suggestions that have helped improve the quality of this paper.

\section{Funding}

No funding was available, this research received no specific grant from any funding agency in the public, commercial, or not-for-profit sectors

Availability of data and materials

Not applicable.

Competing interests

The author declares that he has no competing interest. 


\section{Publisher's Note}

Springer Nature remains neutral with regard to jurisdictional claims in published maps and institutional affiliations.

\section{Received: 20 February 2020 Accepted: 28 May 2020 Published online: 12 June 2020}

\section{References}

1. Mahmoud, W., Ghaleb, A.F., Rawy, E.K., Hassan, H.A.Z., Mosharafa, A.: Numerical solution to a nonlinear, one-dimensional problem of thermoelasticity with volume force and heat supply in a half-space. Arch. Appl. Mech. (2014). https://doi.org/10.1007/s00419-014-0853-y

2. Rawy, E.K., Iskandar, L., Ghaleb, A.F.: Numerical solution for a nonlinear, one-dimensional problem of thermoelasticity. J. Comput. Appl. Math. 100, 53-76 (1998)

3. Abd-Alla, A.N., Ghaleb, A.F., Maugin, G.A.: Harmonic wave generation in nonlinear thermoelasticity. Int. J. Eng. Sci. 32, 1103-1116 (1994)

4. Wu, S.H., Chen, H., Li, W.X.: The local and global existence of the solutions of hyperbolic-parabolic system modeling biological phenomena. Acta Math. Sci. 28B(1), 101-116 (2008)

5. Zheng, S.M.: Nonlinear Parabolic Equations and Hyperbolic-Parabolic Coupled Systems. CRC Press, Boca Raton (1995)

6. Khalifa, M.E.: Existence of almost everywhere solution for nonlinear hyperbolic-parabolic system. Appl. Math. Comput. 145, 569-577 (2003)

7. Sun, D., Manoranjan, V.S., Hong-Ming, Y.: Numerical solutions for a coupled parabolic equations arising induction heating processes. Discrete Contin. Dyn. Syst. 2007, 956-964 (2007)

8. Cui, X:: Galerkin method for some nonlinear coupled problems of hyperbolic and parabolic equations. Numer. Math. J. Chin. Univ. 23, 237-246 (2001)

9. Liu, J.L.: Galerkin methods for the nonlinear coupled problems. Numer. Math. J. Chin. Univ. 4, 260-267 (1982)

10. Liu, X.Z., Cui, X., Sun, J.G.: FDM for multi-dimensional nonlinear coupled system of parabolic and hyperbolic equations. J. Comput. Appl. Math. 186, 432-449 (2006)

11. Sweilam, N.H.: Harmonic wave generation in nonlinear thermoelasticity by variational iteration method and Adomian's method. J. Comput. Appl. Math. 207, 64-72 (2007)

12. Sweilam, N.H., Khader, M.M.: Variational iteration method for one-dimensional nonlinear thermoelasticity. Chaos Solitons Fractals 32, 145-149 (2007)

13. Podlubny, I.: Fractional Differential Equations. Academic Press, San Diego (1999)

14. Herrmann, R.: Fractional Calculus, an Introduction for Physicists. World Scientific, Singapore (2011)

15. Daftardar-Gejij, V., Jafari, H.: Solving a multi-order fractional differential equation using Adomian decomposition. Appl. Math. Comput. 189(1), 541-548 (2007)

16. Ervin, V.J., Roop, J.P.: Variational formulation for the stationary fractional advection dispersion equation. Numer. Methods Partial Differ. Equ. 22, 558-576 (2006)

17. Zeng, F., Liu, F., Li, C., Burrage, K., Turner, I., Anh, V.: A Crank-Nicolson ADI spectral method for a two-dimensional Riesz space fractional nonlinear reaction-diffusion equation. SIAM J. Numer. Anal. 52(6), 2599-2622 (2014)

18. Sweilam, N.H., Abou Hasan, M.M.: Numerical studies for the fractional Schrödinger equation with the quantum Riesz-Feller derivative. Prog. Fract. Differ. Appl. 2(4), 231-245 (2016). https://doi.org/10.18576/pfda/020401

19. Golbabai, A., Sayevand, K.: Analytical modelling of fractional advection-dispersion equation defined in a bounded space domain. Math. Comput. Model. 53, 1708-1718 (2011)

20. Sweilam, N.H., Nagy, A.M., El-Sayed, A.A.: Second kind shifted Chebyshev polynomials for solving space fractional order diffusion equation. Chaos Solitons Fractals 73, 141-147 (2015)

21. Sweilam, N.H., Abou Hasan, M.M.: Numerical approximation of Lévy-Feller fractional diffusion equation via Chebyshev-Legendre collocation method. Eur. Phys. J. Plus 131, 251 (2016). https://doi.org/10.1140/epjp/i2016-16251-y

22. Cenesiz, Y., Keskin, Y., Kurnaz, A.: The solution of the Bagley-Torvik equation with the generalized Taylor collocation method. J. Franklin Inst. 347(2), 452-466 (2010)

23. Das, S.: Analytical solution of a fractional diffusion equation by variational iteration method. Comput. Math. Appl. 57(3), 483-487 (2009)

24. Atangana, A., Cloot, A.H.: Stability and convergence of the space fractional variable-order Schrödinger equation. Adv. Differ. Equ. 2013, 80 (2013). https://doi.org/10.1016/j.jcp.2014.08.015

25. Chen, C.M., Liu, F., Anh, V., Turner, I.: Numerical simulation for the variable-order Galilei invariant advection diffusion equation with a nonlinear source term. Appl. Math. Comput. 217, 5729-5742 (2011)

26. Sun, H.G., Chen, W., Wei, H., Chen, Y.Q.: A comparative study of constant-order and variable-order fractional models in characterizing memory property of systems. Eur. Phys. J. Spec. Top. 193(1), 185-192 (2011)

27. Sweilam, N.H., Khader, M.M., Almarwm, H.M.: Numerical studies for the variable order nonlinear fractional wave equation. Fract. Calc. Appl. Anal. 15(4), 669-683 (2012)

28. Sweliam, N.H., Nagy, A.M., Assiri, T.A., Ali, N.Y.: Numerical simulations for variable-order fractional nonlinear delay differential equations. J. Fract. Calc. Appl. 6(1), 71-82 (2015)

29. Zhuang, P., Liu, F., Anh, V., Turner, I.: Numerical methods for the variable-order fractional advection-diffusion equation with a nonlinear source term. SIAM J. Numer. Anal. 47(3), 1760-1781 (2009)

30. Lin, R., Liu, F., Anh, V., Turner, I.: Stability and convergence of a new explicit finite-difference approximation for the variable-order nonlinear fractional diffusion equation. Appl. Math. Comput. 212, 435-445 (2009)

31. Samko, S.G., Ross, B.: Integration and differentiation to a variable fractional order. Integral Transforms Spec. Funct. 1, 277-300 (1993)

32. Zhao, X., Sun, Z., Em, G.: Second-order approximations for variable order fractional derivatives: algorithms and applications. J. Comput. Phys. (2014). https://doi.org/10.1016/j.jcp.2014.08.015

33. Lorenzo, C.F., Hartley, T.T.: Initialization, conceptualization, and application in the generalized fractional calculus, NASA/TP-1998-208415 (1998)

34. Coimbra, C.F.M.: Mechanics with variable-order differential operators. Ann. Phys. 12, 692-703 (2003)

35. Mickens, R.E.: Exact solutions to a finite-difference model of a nonlinear reaction-advection equation: implications for numerical analysis. Numer. Methods Partial Differ. Equ. 5(4), 313-325 (1989) 
36. Mickens, R.E.: Applications of Nonstandard Finite Difference Schemes. World Scientific, Singapore (2000)

37. Mickens, R.E.: Nonstandard finite difference schemes for differential equations. J. Differ. Equ. Appl. 8(9), 823-847 (2002)

38. Arenas, A.J., González-Parrab, G., Chen-Charpentier, B.M.: Construction of nonstandard finite difference schemes for the SI and SIR epidemic models of fractional order. Math. Comput. Simul. 121, 48-63 (2016)

39. Sweilam, N.H. Almajbri, T.F.: Large stability regions method for the two-dimensional fractional diffusion equation. Prog. Fract. Differ. Appl. 1(2), 123-131 (2015)

40. Mickens, R.E., Washington, T.M.: A note on an NSFD scheme for a mathematical model of respiratory virus transmission. J. Differ. Equ. Appl. 18(3), 525-529 (2010)

41. Zhu, D., Kinoshita, S., Cai, D., Cole, J.B.: Investigation of structural colors in Morpho butterflies using the nonstandard-finite-difference time-domain method: effects of alternately stacked shelves and ridge density. Phys. Rev. E 80(5), 051924 (2009)

42. Moaddy, K., Momani, S., Hashim, I.: The non-standard finite difference scheme for linear fractional PDEs in fluid mechanics. Comput. Math. Appl. 61(4), 1209-1216 (2011)

43. Banerjee, S., Cole, J.B., Yatagai, T.: Calculation of diffraction characteristics of sub wavelength conducting gratings using a high accuracy nonstandard finite-difference time-domain method. Opt. Rev. 12(4), 274-280 (2005)

44. Elsheikh, S., Ouifki, R., Patidar, K.C.: A non-standard finite difference method to solve a model of HIV-malaria co-infection. J. Differ. Equ. Appl. 20(3), 354-378 (2014)

45. Moghadas, S., Alexander, M., Corbett, B.: A non-standard numerical scheme for a generalized Gauss-type predator-prey model. Phys. D, Nonlinear Phenom. 188(1), 134-151 (2004)

46. Moaddy, K., Radwan, A.G., Salama, K.N., Momani, S., Hashim, I.: The fractional-order modeling and synchronization of electrically coupled neuron systems. Comput. Math. Appl. 64(10), 3329-3339 (2012)

47. Moaddy, K., Hashim, I., Momani, S.: Non-standard finite difference schemes for solving fractional-order Rössler chaotic and hyperchaotic systems. Comput. Math. Appl. 62(3), 1068-1074 (2011)

48. Nagy, A., Sweilam, N.H.: An efficient method for solving fractional Hodgkin-Huxley model. Phys. Lett. A 378(30), 1980-1984 (2014)

49. Smith, G.D.: Numerical Solution of Partial Differential Equations: Finite Difference Methods. Oxford Applied Mathematics and Computing Science Series (1985)

50. Sweilam, N.H., Abou Hasan, M.M.: Numerical solutions of a general coupled nonlinear system of parabolic and hyperbolic equations of thermoelasticity. Eur. Phys. J. Plus 132, 212 (2017). https://doi.org/10.1140/epjp/i2017-11484-x

51. Yuste, S.B.: Weighted average finite difference methods for fractional diffusion equations. J. Comput. Phys. 216, 264-274 (2006)

52. Sweilam, N.H., Abou Hasan, M.M.: Numerical simulation for the variable-order fractional Schrödinger equation with the quantum Riesz-Feller derivative. Adv. Appl. Math. Mech. 9(4), 990-1011 (2017). https://doi.org/10.4208/aamm.2015.m1312

53. Jiang, S.: Numerical solution for the Cauchy problem in nonlinear 1-D thermoelasticity. Computing 44, 147-158 (1990)

54. Jiang, S.: An uncoupled numerical scheme for the equations of nonlinear one-dimensional thermoelasticity. J. Comput. Appl. Math. 34, 135-144 (1991)

55. Sweilam, N.H., Khader, M.M.: Exact solutions of some coupled nonlinear partial differential equations using the homotopy perturbation method. Comput. Math. Appl. 58, 2134-2141 (2009)

56. Povstenko, Y.Z.: Fractional Thermoelasticity, Solid Mechanics and Its Applications. Springer, Cham (2015)

57. Povstenko, Y.Z.: Fractional heat conduction equation and associated thermal stress. J. Therm. Stresses $28,83-102$ (2005)

58. Povstenko, Y.Z.: Theory of thermoelasticity based on the space-time-fractional heat conduction equation. Phys. Scr. T $136,014017(2009)$

59. Povstenko, Y.Z.: Theories of thermal stresses based on space-time-fractional telegraph equations. Comput. Math. Appl. 64, 3321-3328 (2012)

60. Lorenzo, C.F., Hartley, T.T.: Variable order and distributed order fractional operators. Nonlinear Dyn. 29, 57-98 (2002)

61. Chechkin, A.V., Gorenflo, R., Sokolov, I.M.: Fractional diffusion in inhomogeneous media. J. Phys. A, Math. Gen. 38 , L679-L684 (2005)

62. Sun, H.G., Chen, W., Wei, H., Chen, Y.Q.: A comparative study of constant-order and variable-order fractional models in characterizing memory property of systems. Eur. Phys. J. Spec. Top. 193(1), 185-192 (2011)

63. Sun, H.G., Chang, A., Zhang, Y., Chen, W.: A review on variable-order fractional differential equations: mathematical foundations, physical models, numerical methods and applications. Fract. Calc. Appl. Anal. 22, 25-59 (2019)

64. Demidovich, B.P., Maron, I.A.: Computational Mathematics, English translation. Mir, Moscow (1987)

65. Smith, G.D.: Numerical Solution of Partial Differential Method, 2nd edn. Oxford University Press, London (1978)

66. Yuste, S.B., Acedo, L.: An explicit finite difference method and a new von Neumann-type stability analysis for fractional diffusion equations. SIAM J. Numer. Anal. 42, 1862-1874 (2005)

67. Yuste, S.B., Quintana-Murillo, J.: A finite difference method with non-uniform timesteps for fractional diffusion equations. Comput. Phys. Commun. 183, 2594-2600 (2012) 